\title{
A Heuristic Prover for Elementary Analysis in Theorema
}

\author{
Tudor Jebelean
}

April 2021

RISC Report Series No. 21-07 


\title{
A Heuristic Prover for Elementary Analysis in Theorema
}

\author{
Tudor Jebelean, RISC-Linz, Austria
}

Abstract. We present the application of certain heuristic techniques for the automation of proofs in elementary analysis. the techniques used are: the S-decomposition method for formulae with alternating quantifiers, quantifier elimination by cylindrical algebraic decomposition, analysis of terms behavior in zero, bounding the $\epsilon$-bounds, semantic simplification of expressions involving absolute value, polynomial arithmetic, usage of equal arguments to arbitrary functions, and automatic reordering of proof steps in order to check the admisibility of solutions to the metavariables. The proofs are very similar to those produced automatically, but they are edited for readability and aspect, and also for inserting the appropriate explanation about the use of the proof techniques. The proofs are: convergence of product of two sequences, continuity of the sum of two functions, uniform continuity of the sum of two functions, uniform continuity of the product of two functions, and continuity of the composition of functions.

\section{Convergence of Product of Two Sequences}

\section{Definitions}

Convergence

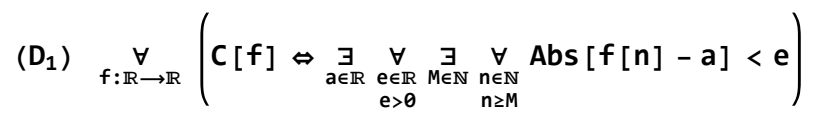

Product of functions

$\left(D_{2}\right) \underset{f_{1}, f_{2}: \mathbb{R} \rightarrow \mathbb{R}}{\forall}\left(\left(f_{1} * f_{2}\right): \mathbb{R} \rightarrow \mathbb{R} \wedge \underset{x \in \mathbb{R}}{\forall}\left(f_{1} * f_{2}\right)[x]=\left(f_{1}[x] * f_{2}[x]\right)\right)$

\section{Product of two convergent sequences is convergent}

Formula to prove

(G) $\underset{\substack{f_{1}, f_{2}: \mathbb{R} \rightarrow \mathbb{R} \\ C\left[f_{1}\right], C\left[f_{2}\right]}}{\forall} C\left[f_{1} * f_{2}\right]$

\section{Proof presentation and explanation of techniques}

For proving (G) we take $f_{1}, f_{2}: \mathbb{R} \rightarrow \mathbb{R}$ arbitrary but fixed, we assume :

$\left(A_{1}\right) C\left[f_{1}\right]$ 


\section{$\left(A_{2}\right) C\left[f_{2}\right]$}

and we prove:

(G) $C\left[f_{1} * f_{2}\right]$

From $\left(A_{1}\right)$, using $f_{1}: \mathbb{R} \rightarrow \mathbb{R}$, by the definition $\left(D_{1}\right)$ we obtain :

$$
\text { (1) } \underset{a \in \mathbb{R}}{\exists} \underset{\substack{e \in \mathbb{R} \\ e>\theta}}{\forall} \underset{M \in \mathbb{N}}{\exists} \underset{\substack{n \in \mathbb{N} \\ n \geq M}}{\forall} \operatorname{Abs}\left[f_{1}[n]-a\right]<e
$$

From $\left(A_{2}\right)$, using $f_{2}: \mathbb{R} \rightarrow \mathbb{R}$, by the definition $\left(D_{1}\right)$ we obtain :

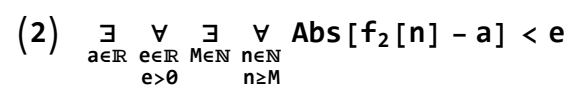

Using $f_{1}, f_{2}: \mathbb{R} \rightarrow \mathbb{R}$, by the definition $\left(D_{2}\right)$ we obtain $\left(f_{1} \star f_{2}\right): \mathbb{R} \rightarrow \mathbb{R}$.

Using $\left(f_{1} \star f_{2}\right): \mathbb{R} \rightarrow \mathbb{R}$, by the definition $\left(D_{1}\right)$, for proving $(G)$ it suffices to prove:

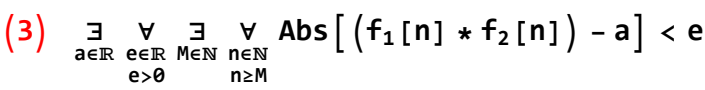

By (1), (2) we can take $a_{1}, a_{2} \in \mathbb{R}$ such that :

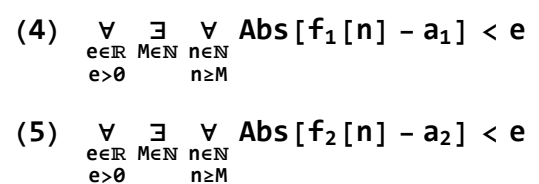

Witness for existential goal : $\mathbf{a}^{?} \rightarrow \mathrm{a}_{1} * \mathrm{a}_{2}$

This is determined by equating to 0 the expressions:

$f_{1}[n]-a_{1}, f_{2}[n]-a_{2},\left(f_{1}[n] * f_{2}[n]\right)-a^{?}$

For proving (3) it is sufficient to prove :

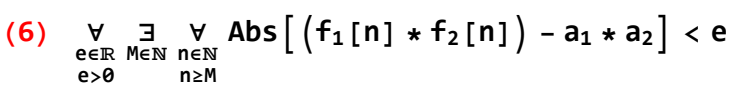

For proving (6) we take $e_{0} \in \mathbb{R}$ arbitrary but fixed, we assume :

(7) $e_{0}>0$

and we prove :

(8) $\underset{M \in \mathbb{N}}{\exists} \underset{\substack{\exists \in \mathbb{N} \\ n \geq M}}{\forall} \operatorname{Abs}\left[\left(f_{1}[n] * f_{2}[n]\right)-a_{1} * a_{2}\right]<e_{\theta}$

Instantiation term for universal assumptions : $\mathrm{e}^{\text {? }}$

First we prove :

(9) $e^{?}>0$

New version of goal (after solving $\mathrm{e}^{\text {? }}$ later):

(9.1) $\operatorname{Min}\left[1, \frac{e_{\ominus}}{1+\operatorname{Abs}\left[a_{1}\right]+\operatorname{Abs}\left[a_{2}\right]}\right]>0$

This follows from (7) using elementary properties of $\mathbb{R}$.

Background test in Mathematica : 


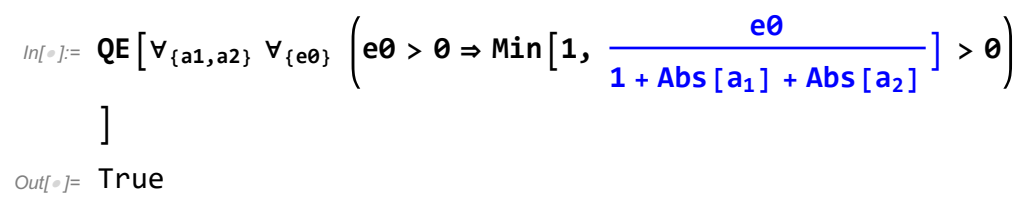

By (10) and (11) we can take $M_{1}, M_{2} \in \mathbb{N}$ such that:

(12) $\underset{\substack{n \in \mathbb{N} \\ n \geq M_{1}}}{\forall} A b s\left[f_{1}[n]-a_{1}\right]<e^{?}$

(13) $\underset{\substack{n \in \mathbb{N} \\ n \geq M_{2}}}{\forall} A b s\left[f_{2}[n]-a_{2}\right]<e^{?}$

Witness for existential goal : M?

In order to prove (8) it suffices to prove:

(14) $\underset{\substack{n \in \mathbb{N} \\ n \geq M^{2}}}{\forall} \operatorname{Abs}\left[\left(f_{1}[n] * f_{2}[n]\right)-a_{1} * a_{2}\right]<e_{\ominus}$

For proving (14) we take $n_{0} \in \mathbb{N}$ arbitrary but fixed, we assume :

(15) $n_{\ominus} \geq M^{?}$

and we prove :

(16) Abs $\left[\left(f_{1}\left[n_{\ominus}\right] * f_{2}\left[n_{\ominus}\right]\right)-a_{1} * a_{2}\right]<e_{\ominus}$

Instantiation term for universal assumptions : $n^{\text {? }}$

Solution by equal terms under unknown function $: n^{?} \rightarrow n_{\ominus}$

(12) $\underset{\substack{n \in \mathbb{N} \\ n \geq M_{1}}}{\forall} \operatorname{Abs}\left[f_{1}[n]-a_{1}\right]<e^{?}$

In the assumptions the variable n occurs under the "unknown" (was universally quantified) function symbol $f_{1}$.

In the goal (16) we have $f_{1}\left[n_{\ominus}\right]$.

Later: Replace terms with unknown functions by symbols.

First we prove :

(17) $\left(n_{\ominus} \geq M_{1}\right) \wedge\left(n_{\ominus} \geq M_{2}\right)$

Solution for witness for existential goal (14) : M? $\rightarrow$ Max [M1, M2]

We prove the conditions of assumptions (12) and (13) using the condition (15) of goal (14). Use quantifier elimination: 
4 | techreport.nb

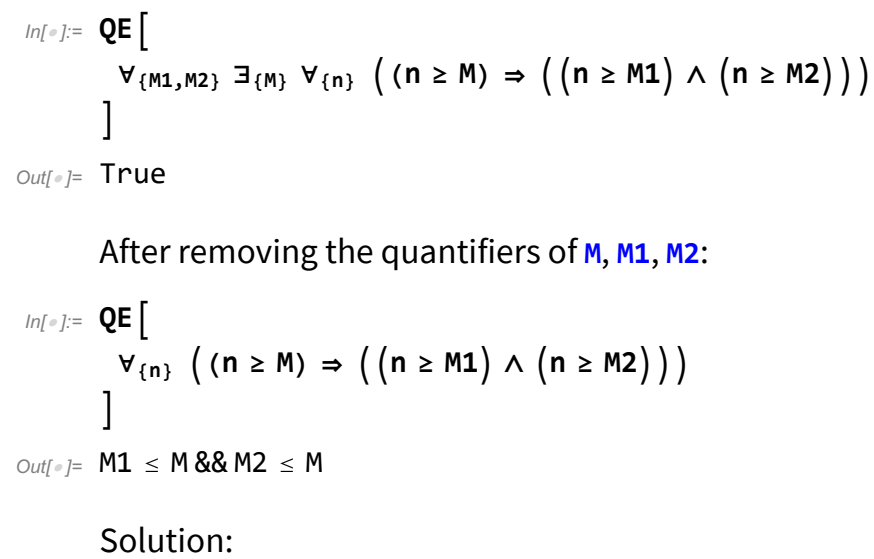

Solution:

$M \rightarrow \operatorname{Max}[\mathrm{M} 1, \mathrm{M} 2]$

New version of assumption :

(15.1) $\mathrm{n}_{0} \geq \operatorname{Max}[M 1, M 2]$

The goal (17) follows from (15) using elementary properties of $\mathbb{R}$.

$\ln [\cdot]:=\mathbf{Q E}[$

$\forall_{\{n\}}((n \geq \operatorname{Max}[M 1, M 2]) \Rightarrow((n \geq M 1) \wedge(n \geq M 2)))$

]

$16 \mathrm{~ms}(16+0)$

Out $[0]=$ True

Using (17), from (12) and (13) we obtain :

(18) Abs $\left[f_{1}\left[n_{\ominus}\right]-a_{1}\right]<e^{\text {? }}$

(19) Abs $\left[f_{2}\left[n_{\ominus}\right]-a_{2}\right]<e^{\text {? }}$

\section{Algebraic manipulations}

These steps are performed in the background, they are not visible in the final proof.

In order to obtain a polynomial problem, we replace each term whose head is an unknown function by a new symbol:

(In the parallel quantified formula, the new symbol will be quantified like $\boldsymbol{n}_{\mathbf{0}}$ )

$f_{1}\left[n_{\ominus}\right] \rightarrow x_{1} \quad f_{2}\left[n_{\ominus}\right] \rightarrow x_{2}$

New assumptions:

(18.1) Abs $\left[x_{1}-a_{1}\right]<e^{?}$

(19.1) Abs $\left[x_{2}-a_{2}\right]<e^{\text {? }}$

New goal:

(16.1) Abs $\left[\left(x_{1} * x_{2}\right)-a_{1} * a_{2}\right]<e_{0}$

Solution for instantiation term for universal assumptions : $\mathbf{e}^{\text {? }} \rightarrow \frac{\mathbf{e} \boldsymbol{0}}{\mathbf{1}+\mathbf{A b s}\left[\mathbf{a}_{\mathbf{1}}\right]+\mathbf{A b s}\left[\mathbf{a}_{\mathbf{2}}\right]}$

Method: Use simplification of expressions and solving

In (18.1) and (19.1) replace argument of Abs by new symbols: 
$x_{1}-a_{1}=y_{1} \quad x_{2}-a_{2}=y_{2}$

Solve for the quantified variables:

$x_{1}=y_{1}+a_{1} \quad x_{2}=y_{2}+a_{2}$

Replace in assumptions and goal:

(18.2) Abs $\left[y_{1}\right]<e^{?}$

(19.2) Abs $\left[y_{2}\right]<e^{?}$

$(16.2)$ Abs $\left[\left(y_{1}+a_{1}\right) *\left(y_{2}+a_{2}\right)-\left(a_{1} * a_{2}\right)\right]<e_{\ominus}$

Straightforward computation:

$\ln [\cdot]:=$ Expand $\left[\left(y_{1}+a_{1}\right) *\left(y_{2}+a_{2}\right)-\left(a_{1} * a_{2}\right)\right]$

Out[o]= $a_{2} y_{1}+a_{1} y_{2}+y_{1} y_{2}$

(16.3) Abs $\left[a_{2} y_{1}+a_{1} y_{2}+y_{1} y_{2}\right]<e_{0}$

Rewrite LHS by increasing it (semantic simplification of expressions involving absolute value):

Abs $\left[a_{2} y_{1}+a_{1} y_{2}+y_{1} y_{2}\right] \rightarrow$ Abs $\left[a_{2} y_{1}\right]+A b s\left[a_{1} y_{2}\right]+A b s\left[y_{1} y_{2}\right] \rightarrow$

Abs $\left[a_{2}\right]$ Abs $\left[y_{1}\right]+$ Abs $\left[a_{1}\right]$ Abs $\left[y_{2}\right]+$ Abs $\left[y_{1}\right]$ Abs $\left[y_{2}\right] \rightarrow$

Abs $\left[a_{2}\right] e^{?}+\operatorname{Abs}\left[a_{1}\right] e^{?}+e^{?} e^{?} \rightarrow$ Abs $\left[a_{2}\right] e^{?}+A b s\left[a_{1}\right] e^{?}+e^{?} \rightarrow\left(\right.$ Abs $\left.\left[a_{2}\right]+A b s\left[a_{1}\right]+1\right) * e^{?}$

(Assume $e^{?} \leq 1$ )

Solve for $e^{?}$ :

$\left(\operatorname{Abs}\left[a_{2}\right]+\operatorname{Abs}\left[a_{1}\right]+1\right) * e^{?}=e \theta$

New version of assumptions:

(20) Abs $\left[x_{1}-a_{1}\right]<\operatorname{Min}\left[1, \frac{e_{\theta}}{\operatorname{Abs}\left[a_{2}\right]+\operatorname{Abs}\left[a_{1}\right]+1}\right]$
(21) Abs $\left[x_{2}-a_{2}\right]<\operatorname{Min}\left[1, \frac{e_{\theta}}{\operatorname{Abs}\left[a_{2}\right]+\operatorname{Abs}\left[a_{1}\right]+1}\right]$

The goal (22) follows from the assumptions (20) and (21) using elementary properties of $\mathbb{R}$.

\section{Finalization of the proof}

In the final proof the corresponding step is announced as :

By elementary properties of $\mathbb{R}$ we transform the goal (16) into :

(20) Abs $\left[a_{2} *\left(x_{1}-a_{1}\right)+a_{1} *\left(x_{2}-a_{2}\right)+\left(x_{1}-a_{1}\right)\left(x_{2}-a_{2}\right)\right]<e_{\ominus}$

(Remark : this corresponds to the hidden goal (16.3).)

By (18), (19), and elementary properties of $\mathbb{R}$, assuming $e^{?}<1$ we have:

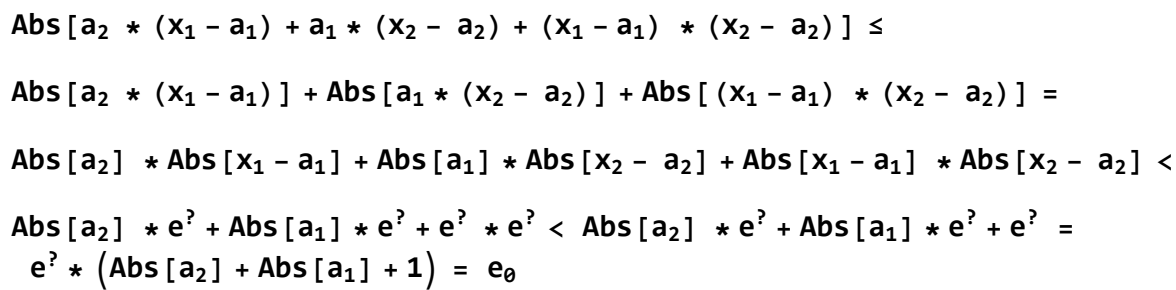

which proves the goal. 


\section{Continuity of Sum of Two Functions}

\section{Definitions}

Continuity

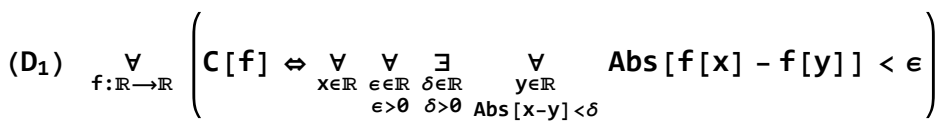

Sum of functions

$\left(D_{2}\right) \underset{f_{1}, f_{2}: \mathbb{R} \rightarrow \mathbb{R}}{\forall}\left(\left(f_{1}+f_{2}\right): \mathbb{R} \rightarrow \mathbb{R} \wedge \underset{x \in \mathbb{R}}{\forall}\left(f_{1}+f_{2}\right)[x]=\left(f_{1}[x]+f_{2}[x]\right)\right)$

\section{Sum of continuous functions is continuous}

Formula to prove

(G) $\underset{\substack{f_{1}, f_{2}: \mathbb{R} \rightarrow \mathbb{R} \\ c\left[f_{1}\right], C\left[f_{2}\right]}}{\forall} C\left[f_{1}+f_{2}\right]$

\section{Proof presentation}

For proving (G) we take $f_{1}, f_{2}: \mathbb{R} \rightarrow \mathbb{R}$ arbitrary but fixed, we assume :

$\left(A_{1}\right) C\left[f_{1}\right]$

$\left(A_{2}\right) \quad C\left[f_{2}\right]$

and we prove:

(G) $C\left[f_{1}+f_{2}\right]$

From $\left(A_{1}\right)$, using $f_{1}: \mathbb{R} \rightarrow \mathbb{R}$, by the definition $\left(D_{1}\right)$ we obtain :

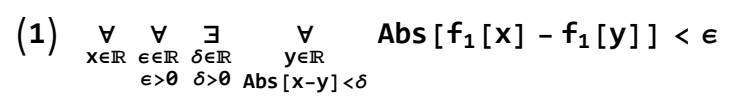

From $\left(A_{2}\right)$, using $f_{2}: \mathbb{R} \rightarrow \mathbb{R}$, by the definition $\left(D_{1}\right)$ we obtain :

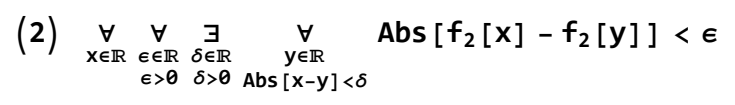

Using $f_{1}, f_{2}: \mathbb{R} \rightarrow \mathbb{R}$, by the definition $\left(D_{2}\right)$ we obtain $\left(f_{1}+f_{2}\right): \mathbb{R} \rightarrow \mathbb{R}$.

Using $\left(f_{1}+f_{2}\right): \mathbb{R} \rightarrow \mathbb{R}$, by the definition $\left(D_{1}\right)$, for proving $(G)$ it suffices to prove:

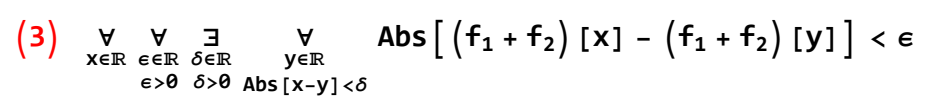

Using $f_{1}, f_{2}: \mathbb{R} \rightarrow \mathbb{R}, x, y \in \mathbb{R}$, by the definition $\left(D_{2}\right)$, for proving (3) it suffices to prove:

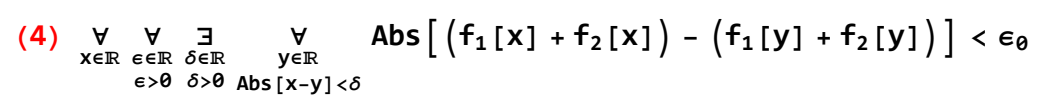


For proving (4) we take $x_{0} \in \mathbb{R}$ arbitrary but fixed and we prove :

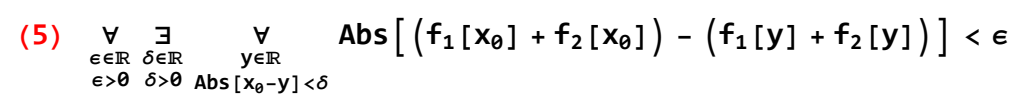

Using $x_{0} \in \mathbb{R}$, we instantiate (1) and we obtain :

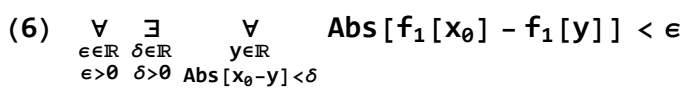

Using $x_{0} \in \mathbb{R}$, we instantiate (2) and we obtain :

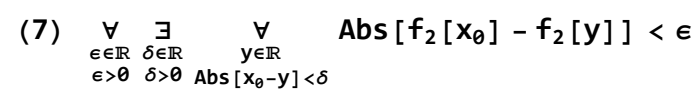

For proving (5) we take $\epsilon_{0} \in \mathbb{R}$ arbitrary but fixed, we assume :

(8) $\epsilon_{\theta}>0$

and we prove :

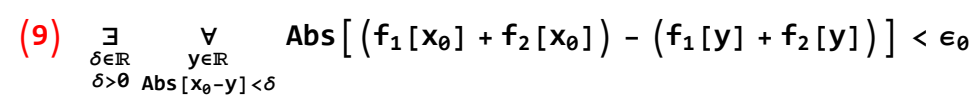

We consider :

$\epsilon_{1}=\epsilon_{\ominus} / 2$

First we prove $\epsilon_{1} \in \mathbb{R}$ and:

(10) $\epsilon_{1}>0$

These follow from $\epsilon_{0} \in \mathbb{R}$, (8), and elementary properties of $\mathbb{R}$.

Using $\epsilon_{1} \in \mathbb{R}$ and (10), we instantiate (6) and we obtain :

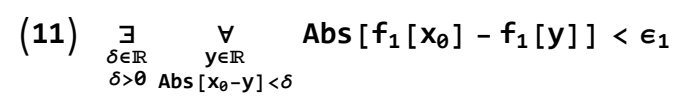

Using $\epsilon_{1} \in \mathbb{R}$ and (10), we instantiate (7) and we obtain :

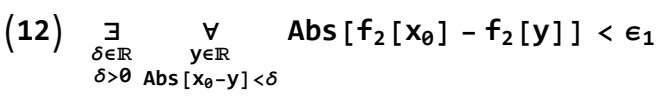

By (11) we can take $\delta_{1} \in \mathbb{R}$, such that:

(13) $\delta_{1}>\theta \wedge \underset{\substack{y \in \mathbb{R} \\ \operatorname{Abs}\left[x_{\theta}-y\right]<\delta_{1}}}{\forall} \operatorname{Abs}\left[f_{1}\left[x_{\theta}\right]-f_{1}[y]\right]<\epsilon_{1}$

By (12) we can take $\delta_{2} \in \mathbb{R}$ such that :

(14) $\delta_{2}>\theta \wedge \underset{\substack{y \in \mathbb{R} \\ \operatorname{Abs}\left[x_{\theta}-y\right]<\delta_{2}}}{\forall} \operatorname{Abs}\left[f_{2}\left[x_{\theta}\right]-f_{2}[y]\right]<\epsilon_{1}$

We consider :

$\delta_{\theta}=\operatorname{Min}\left[\delta_{1}, \delta_{2}\right]$

First we prove $\delta_{0} \in \mathbb{R}$ and:

(15) $\delta_{\theta}>0$

These follow from $\delta_{1}, \delta_{2} \in \mathbb{R}$, (13.1), (14.1) and elementary properties of $\mathbb{R}$.

Using $\delta_{0} \in \mathbb{R}$ and (15), in order to prove (9) it suffices to prove: 


$$
\underset{\substack{y \in \mathbb{R} \\ \forall}\left[x_{\theta}-y\right]<\delta_{\theta}}{\forall} \operatorname{Abs}\left[\left(f_{1}\left[x_{\theta}\right]+f_{2}\left[x_{\theta}\right]\right)-\left(f_{1}[y]+f_{2}[y]\right)\right]<\epsilon_{\theta}
$$

For proving (16) we take $y_{0} \in \mathbb{R}$ arbitrary but fixed, we assume :

(17) Abs $\left[x_{\theta}-y_{\theta}\right]<\delta_{\theta}$

and we prove:

(18) Abs $\left[\left(f_{1}\left[x_{\ominus}\right]+f_{2}\left[x_{\ominus}\right]\right)-\left(f_{1}\left[y_{\ominus}\right]+f_{2}\left[y_{\ominus}\right]\right)\right]<\epsilon_{\ominus}$

First we prove :

(19) Abs $\left[\mathrm{x}_{\theta}-\mathrm{y}_{\theta}\right]<\delta_{1} \wedge$ Abs $\left[\mathrm{x}_{\theta}-\mathrm{y}_{\theta}\right]<\delta_{2}$

This follows from $x_{0}, y_{0}, \delta_{0}, \delta_{1}, \delta_{2} \in \mathbb{R},(17)$ and elementary properties of $\mathbb{R}$.

Using $y_{0} \in \mathbb{R}$ and (19.1), we instantiate (13.2) and we obtain:

(20) Abs $\left[f_{1}\left[x_{\theta}\right]-f_{1}\left[y_{\theta}\right]\right]<\epsilon_{1}$

Using $y_{0} \in \mathbb{R}$ and (19.2), we instantiate (14.2) we obtain:

(21) Abs $\left[f_{2}\left[x_{\theta}\right]-f_{2}\left[y_{\theta}\right]\right]<\epsilon_{1}$

Using elementary properties of $\mathbb{R}$ we transform (18) into:

(22) Abs $\left[\left(f_{1}\left[x_{\theta}\right]-f_{1}\left[y_{\theta}\right]\right)+\left(f_{2}\left[x_{\theta}\right]-f_{2}\left[y_{\theta}\right]\right)\right]<\epsilon_{\theta}$

Using elementary properties of $\mathbb{R}$, from (20) and (21) we obtain:

$$
\begin{aligned}
& \operatorname{Abs}\left[\left(f_{1}\left[x_{\theta}\right]-f_{1}\left[y_{\theta}\right]\right)+\left(f_{2}\left[x_{\theta}\right]-f_{2}\left[y_{\ominus}\right]\right)\right] \leq(* \text { by Abs }+*) \\
\leq & \operatorname{Abs}\left[f_{1}\left[x_{\ominus}\right]-f_{1}\left[y_{\theta}\right]+\operatorname{Abs}\left[f_{2}\left[x_{\ominus}\right]-f_{2}\left[y_{\theta}\right]<(* \text { by }(2 \theta),(21) *)\right.\right. \\
< & \epsilon_{1}+\epsilon_{1}=\epsilon_{\theta} / 2+\epsilon_{\theta} / 2=\epsilon_{\theta}
\end{aligned}
$$

which proves the goal.

\section{Proof discovery}

Brown text constitutes comment and is not part of the proof.

[1] Inference rule "arbitrary but fixed" : For proving an universally quantified formula with type declarations and conditions, use arbitrary but fixed constants instead of the quantified variables, assume they satisfy the type declarations and the conditions, and prove the unquantified formula.

For proving (G) we take $f_{1}, f_{2}: \mathbb{R} \rightarrow \mathbb{R}$ arbitrary but fixed, we assume :

$\left(A_{1}\right) \quad\left[f_{1}\right]$

\section{$\left(A_{2}\right) C\left[f_{2}\right]$}

and we prove:

(G) $C\left[f_{1}+f_{2}\right]$

Expanding the assumptions and the goal by definition. When instantiating the definition, check the type declaration and the conditions for the instantiating term.

From $\left(A_{1}\right)$, using $f_{1}: \mathbb{R} \rightarrow \mathbb{R}$, by the definition $\left(D_{1}\right)$ we obtain :

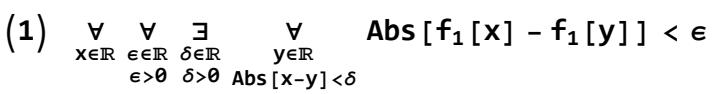


From $\left(A_{2}\right)$, using $f_{2}: \mathbb{R} \rightarrow \mathbb{R}$, by the definition $\left(D_{1}\right)$ we obtain :

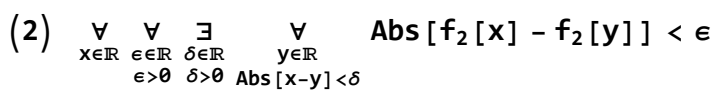

Using $f_{1}, f_{2}: \mathbb{R} \rightarrow \mathbb{R}$, by the definition $\left(D_{2}\right)$ we obtain $\left(f_{1}+f_{2}\right): \mathbb{R} \rightarrow \mathbb{R}$.

Using $\left(f_{1}+f_{2}\right): \mathbb{R} \rightarrow \mathbb{R}$, by the definition $\left(D_{1}\right)$ in order to prove $(G)$ it suffices to prove:

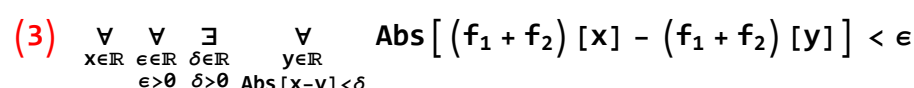

[2] Inference rule "rewriting by equality": A subterm which matches the LHS of a universally quantified equality is replaced by the instantiated RHS of the equality, after checking that the instantiating terms satisfy the type declarations and the conditions of the universal quantifiers. (The rule can be applied similarly for using the equality from right to left.)

Using $f_{1}, f_{2}: \mathbb{R} \rightarrow \mathbb{R}, x, y \in \mathbb{R}$, by $\left(D_{2}\right)$, for proving (3) it suffices to prove:

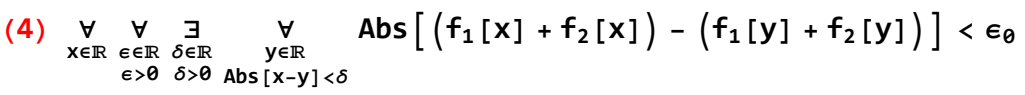

[3] Strategy "S-decomposition": When the assumptions and the goal have identical alternating quantifiers, eliminate quantifiers in parallel step by step, using a block of inference rules for each quantifier.

[4] "S-decomposition" block of inferences for the universal quantifier: When the assumptions and the goal are universally quantified, use first the rule "arbitrary but fixed"[1] for the goal, and then the rule "instantiation"[5] for the assumptions.

Inference rule "arbitrary but fixed" [1].

For proving (4) we take $x_{0} \in \mathbb{R}$ arbitrary but fixed and we prove :

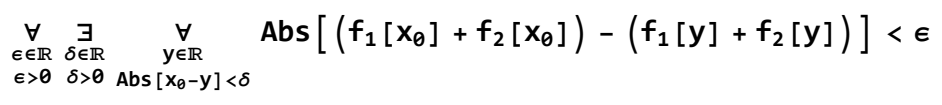

[5] Inference rule "instantiation": When an assumption is universally quantified, instantiate it with an appropriate ground term, after checking that the term satisfies the type declaration and the condition. In this case we instantiate (1).

[6] Method "equal arguments to arbitrary functions": The appropriate instantiation term needed as an argument for an arbitrary function occurring in an assumption must equal a term which already occurs in the proof as the argument of this function occurring in the goal (because otherwise it will not be possible for the proof to succeed). In this case we choose $x_{0}$ because $f_{1}\left[x_{0}\right]$ occurs in the goal.

Using $x_{0} \in \mathbb{R}$, we instantiate (1) and we obtain :

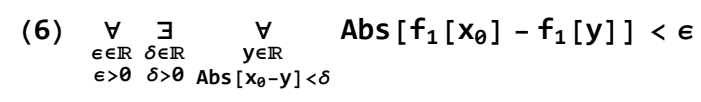

Inference rule "instantiation" [5]: we instantiate (2).

Method "equal arguments to arbitrary functions"[6]: we choose $x_{0}$ because $f_{2}\left[x_{0}\right]$ occurs in the goal.

Using $x_{0} \in \mathbb{R}$, we instantiate (2) and we obtain :

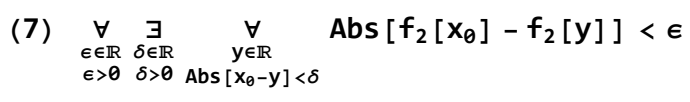


"S-decomposition" block of inferences for the universal quantifier [4].

Inference rule "arbitrary but fixed"[1].

For proving (5) we take $\epsilon_{0} \in \mathbb{R}$ arbitrary but fixed, we assume :

(8) $\epsilon_{\theta}>0$

and we prove :

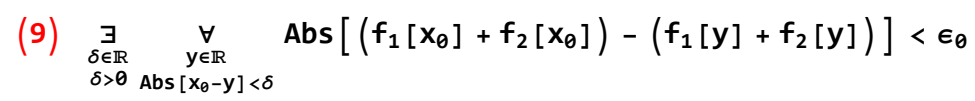

Inference rule "instantiation" [5]: we instantiate (6) and (7).

[7] Method: "meta-variables": in order to find an appropriate term for the instantiation of an universal assumption (or the witness term for an existential goal), use a new meta-variable instead of the term (can be seen as a name for the unknown term) and continue the proof. Later in the proof find the appropriate term ("solve" the meta-variable), and check that the solution is admissible -contains only constants which have been already present in the proof at the moment when the meta-variable was introduced. Also check that the value satisfies the type declaration and the condition of the quantifier. In this case we use the meta-variable $\epsilon_{1}$ and the solution is found at step (23):

$\epsilon_{1}=\epsilon_{\ominus} / 2$

First we prove $\epsilon_{1} \in \mathbb{R}$ and:

(10) $\epsilon_{1}>0$

These follow from $\epsilon_{0} \in \mathbb{R}$, (8), and elementary properties of $\mathbb{R}$.

Using $\epsilon_{1} \in \mathbb{R}$ and (10), we instantiate (6) and we obtain :

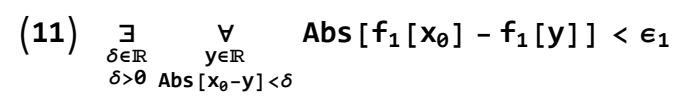

Using $\epsilon_{1} \in \mathbb{R}$ and (10), we instantiate (7) and we obtain :

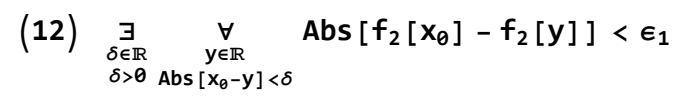

[8] "S-decomposition" block of inferences for the existential quantifier: When the assumptions and the goal are existentially quantified, use first the rule "take such a" [9] for the assumptions, and then the rule "witness"[11] for the goal.

[9] Inference rule "take such a": When an assumption is existentially quantified, use new constants instead of the quantified variables, assume they satisfy the type declarations and the conditions, and use (as assumption) the unquantified formula.

By (11) we can take $\delta_{1} \in \mathbb{R}$, such that :

(13) $\delta_{1}>\theta \wedge \underset{\substack{y \in \mathbb{R} \\ \operatorname{Abs}[x-y]<\delta_{1}}}{\forall} \operatorname{Abs}\left[f_{1}\left[x_{\theta}\right]-f_{1}[y]\right]<\epsilon_{1}$

By (12) we can take $\delta_{2} \in \mathbb{R}$ such that :
(14) $\delta_{2}>0$

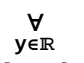
$\operatorname{Abs}\left[f_{2}\left[x_{\theta}\right]-f_{2}[y]\right]<\epsilon_{1}$
Abs $\left[x_{\theta}-y\right]<\delta_{2}$ 
[10] Inference rule "split assumed conjunction": The conjuncts of an assumption can be used later in the proof as individual assumptions. In this case they will be used later as (13.1), (13.2), (14.1), and (14.2).

[11] Inference rule "witness": When goal is existentially quantified, use an appropriate ground term as witness instead of the existential variable, check that the term satisfies the type declaration and the condition, and then prove the unquantified formula. In this case the goal is (9).

Method: "meta-variables". We use the meta-variable $\delta_{0}$ for the witness term. The solution is found at step (19):

$\delta_{\theta}=\operatorname{Min}\left[\delta_{1}, \delta_{2}\right]$

First we prove $\delta_{0} \in \mathbb{R}$ and:

(15) $\delta_{\theta}>0$

These follow from $\delta_{1}, \delta_{2} \in \mathbb{R}$, (13.1), (14.1) and elementary properties of $\mathbb{R}$.

Using $\delta_{0} \in \mathbb{R}$ and (15), in order to prove (9) it suffices to prove:

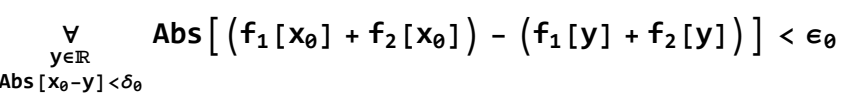

"S-decomposition" block of inferences for the universal quantifier [4].

Inference rule "arbitrary but fixed" [1]

For proving (16) we take $y_{0} \in \mathbb{R}$ arbitrary but fixed, we assume :

(17) Abs $\left[x_{\theta}-y_{\theta}\right]<\delta_{\theta}$

and we prove:

(18) $\operatorname{Abs}\left[\left(f_{1}\left[x_{\theta}\right]+f_{2}\left[x_{\theta}\right]\right)-\left(f_{1}\left[y_{\theta}\right]+f_{2}\left[y_{\theta}\right]\right)\right]<\epsilon_{\theta}$

Inference rule "instantiation" [5]: we instantiate (13.2) and (14.2).

Method "equal arguments to arbitrary functions" [6]: we choose $y_{0}$ because $f_{2}\left[y_{0}\right]$ occurs in the goal.

First we prove :

(19) Abs $\left[x_{\theta}-y_{\theta}\right]<\delta_{1} \wedge$ Abs $\left[x_{\theta}-y_{\theta}\right]<\delta_{2}$

[12] Method "replace equal ground terms by constants": when the same ground term occurs in several places in the formulae composing the current proof situation, we replace it by a new constant. In this case the current proof situation consists in assumption (17) and goal (19). We replace Abs $\left[\mathrm{x}_{\theta}-\mathrm{y}_{\theta}\right]$ by $\mathrm{p}$ and we transform the proof situation into:

$(17.1) \mathrm{p}<\delta_{0}$

(19.1) $\mathrm{p}<\delta_{1} \wedge \mathrm{p}<\delta_{2}$

[13] Method "solve meta-variable": use for the meta-variable a value which makes a simple proof situation to succeed, then check the appropriate conditions - see [7]. In this case the solution is $\operatorname{Min}\left[\delta_{1}, \delta_{2}\right]$. Now we go back to the proof step (15) and check that the constants $\delta_{1}, \delta_{2}$ are already present in the proof at this step, and that the solution satisfies the type declaration $(\delta \in \mathbb{R})$ and the condition $(\delta>0)$ of the main quantifier of goal (9).

In the proof presentation this sub-proof is hidden and we will just write as argument for (19): 
This follows from $x_{0}, y_{0}, \delta_{\theta}, \delta_{1}, \delta_{2} \in \mathbb{R},(17)$ and elementary properties of $\mathbb{R}$.

Inference rule "split assumed conjunction"[10]. Since (19) has been proved, we can use it now as an assumption, and split it into (19.1) and (19.2).

Using (19.1), from (13.2) we obtain:

(20) Abs $\left[f_{1}\left[x_{\theta}\right]-f_{1}\left[y_{\theta}\right]\right]<\epsilon_{1}$

Using (19.2), from (14.2) we obtain:

(21) Abs $\left[f_{2}\left[x_{\theta}\right]-f_{2}\left[y_{\theta}\right]\right]<\epsilon_{1}$

At this moment all quantifiers are eliminated by the "S-decomposition" strategy [3]. The proof situation contains only ground formulae: assumptions (20), (21) and goal (18).

Method "replace equal ground terms by constants" [12]. We replace $f_{1}\left[x_{\ominus}\right], f_{2}\left[x_{\ominus}\right], f_{1}\left[y_{\theta}\right]$, $f_{2}\left[y_{0}\right]$ by $x_{1}, x_{2}, y_{1}, y_{2}$, respectively. The new proof situation is:

(20.1) Abs $\left[x_{1}-y_{1}\right]<\epsilon_{1}$

(21.1) Abs $\left[x_{2}-y_{2}\right]<\epsilon_{1}$

(18.1) Abs $\left[\left(x_{1}+x_{2}\right)-\left(y_{1}+y_{2}\right)\right]<\epsilon_{\theta}$

[14] Method "use constants for terms with known behaviour": replace certain ground terms occurring in the assumptions by constants, and then use equation solving, substitution, and computation in order to transform the corresponding subterms in the goal. In this case we replace $x_{1}-y_{1}$ and $\mathrm{x}_{2}-\mathrm{y}_{2}$ by $a_{1}, a_{2}$, respectively.

$\ln [\cdot]:=\operatorname{EqS}=\left\{\mathbf{a}_{1}==\mathbf{x}_{1}-\mathbf{y}_{1}, \mathrm{a}_{2}=\mathbf{x _ { 2 }}-\mathbf{y}_{2}\right\} ;$

In order to update the goal we eliminate $y_{1}, y_{2}$ because they correspond to constants introduced later in the proof - in this way we have better chances that the solution is admissible.

$\ln [\cdot]:=$ Sols $=$ First $\left[\right.$ Solve $\left[\right.$ Eqs, $\left.\left.\left\{\mathbf{y}_{1}, \mathbf{y}_{2}\right\}\right]\right]$

out $[\cdot]=\left\{\mathbf{y}_{1} \rightarrow-\mathrm{a}_{1}+\mathrm{x}_{1}, \mathrm{y}_{2} \rightarrow-\mathrm{a}_{2}+\mathrm{x}_{2}\right\}$

$\ln [\cdot]:=$ Expand $\left[\left(\mathbf{x}_{1}+\mathbf{x}_{2}\right)-\left(\mathbf{y}_{1}+\mathbf{y}_{2}\right) /\right.$. Sols $]$

Out[o]= $\mathrm{a}_{1}+\mathrm{a}_{2}$

The new proof situation is:

(20.2) Abs $\left[a_{1}\right]<\epsilon_{1}$

(21.2) Abs $\left[a_{2}\right]<\epsilon_{1}$

(18.2) Abs $\left[a_{1}+a_{2}\right]<\epsilon_{\theta}$

In the proof presentation this process is hidden, we just write the expression in (18.2) by substituting back to the original terms:

Using elementary properties of $\mathbb{R}$ we transform (18) into:

(22) $\operatorname{Abs}\left[\left(f_{1}\left[x_{\theta}\right]-f_{1}\left[y_{\theta}\right]\right)+\left(f_{2}\left[x_{\theta}\right]-f_{2}\left[y_{\theta}\right]\right)\right]<\epsilon_{\theta}$

[15] Method "rewrite goal by inequalities": in order to prove an inequality, replace subterms by bigger subterms.

Abs $\left[a_{1}+a_{2}\right] \leq(*$ by Abs $+*)$ 
Abs $\left[a_{1}\right]+$ Abs $\left[a_{2}\right]<\left(*\right.$ by $\left.\left(20^{\prime}\right),\left(21^{\prime}\right) *\right)$

$\epsilon_{1}+\epsilon_{1}$

From the transformations above we have the new assumption:

(23) Abs $\left[a_{1}+a_{2}\right]<2 * \epsilon_{1}$

Method "replace equal ground terms by constants" [12]. We transform (23) and the current goal $(18$ "):

(23.1) $\mathrm{c}<2 * \epsilon_{1}$

$(18.3) c<\epsilon_{\theta}$

Method "solve meta-variable"[13]. In order to succeed in the proof situation above, we find $\in_{1}$ :

$\epsilon_{1}=\epsilon_{0} / 2$

Now we go back to step (10) and check that the constant $\mathbf{e}_{\ominus}$ occurring in the solving term is already present in the proof, and that the type declaration and the conditions of the universal quantifiers in (6) and (7) are satisfied.

Now the proof is finished because the main goal was proven (by an appropriate choice of a solution for the meta-variable).

In the proof presentation this process is hidden, instead the following argument is given, which is produced by substituting in the expressions above the original ground terms:

Using elementary properties of $\mathbb{R}$, from (20) and (21) we obtain:

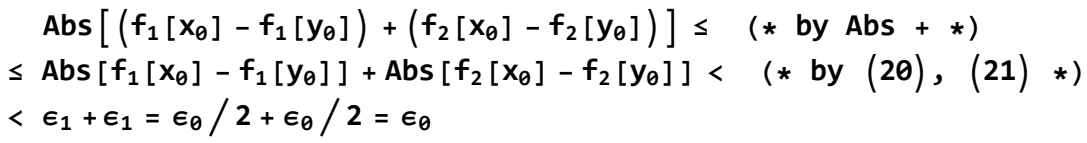

which proves the goal.

\section{Uniform Continuity of Sum of Two Functions}

\section{Definitions}

Uniform continuity

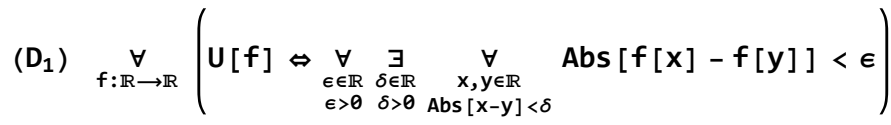

Sum of functions

$\left(D_{2}\right) \underset{f_{1}, f_{2}: \mathbb{R} \rightarrow \mathbb{R}}{\forall}\left(\left(f_{1}+f_{2}\right): \mathbb{N} \rightarrow \mathbb{R} \wedge \underset{x \in \mathbb{R}}{\forall}\left(f_{1}+f_{2}\right)[x]=\left(f_{1}[x]+f_{2}[x]\right)\right)$ 


\section{Sum of uniformly continuous functions is uniformly continuous}

Formula to prove

(G) $\underset{\substack{f_{1}, f_{2}: \mathbb{R} \rightarrow \mathbb{R} \\ U\left[f_{1}\right], U\left[f_{2}\right]}}{\forall} U\left[f_{1}+f_{2}\right]$

\section{Proof presentation}

For proving $(G)$ we take $f_{1}, f_{2}: \mathbb{R} \rightarrow \mathbb{R}$ arbitrary but fixed, we assume :

$\left(A_{1}\right) \cup\left[f_{1}\right]$

$\left(A_{2}\right) \cup\left[f_{2}\right]$

and we prove:

(G) $U\left[f_{1}+f_{2}\right]$

From $\left(A_{1}\right)$, using $f_{1}: \mathbb{R} \rightarrow \mathbb{R}$, by the definition $\left(D_{1}\right)$ we obtain :

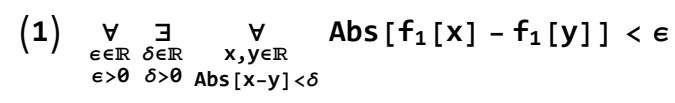

From $\left(A_{2}\right)$, using $f_{2}: \mathbb{R} \rightarrow \mathbb{R}$, by the definition $\left(D_{1}\right)$ we obtain :

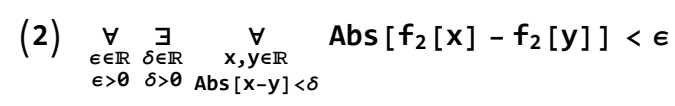

Using $f_{1}, f_{2}: \mathbb{R} \rightarrow \mathbb{R}$, by the definition $\left(D_{2}\right)$ we obtain $\left(f_{1}+f_{2}\right): \mathbb{N} \rightarrow \mathbb{R}$.

Using $\left(f_{1}+f_{2}\right): \mathbb{N} \rightarrow \mathbb{R}$, by the definition $\left(D_{1}\right)$, for proving $(G)$ it suffices to prove:

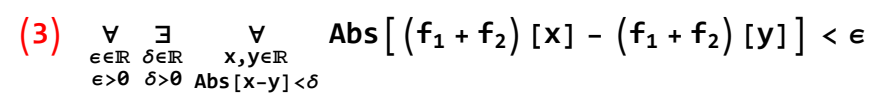

Using $f_{1}, f_{2}: \mathbb{R} \rightarrow \mathbb{R}, x, y \in \mathbb{R}$, by the definition $\left(D_{2}\right)$, for proving (3) it suffices to prove:

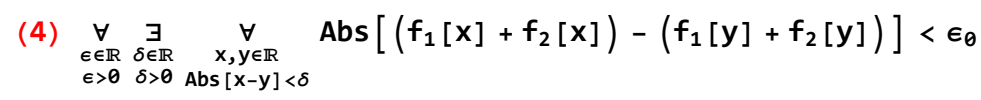

For proving (4) we take $\epsilon_{0} \in \mathbb{R}$ arbitrary but fixed, we assume :

(5) $\epsilon_{\theta}>0$

and we prove:

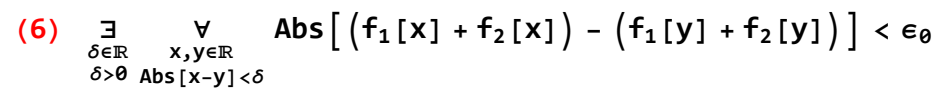

We consider :

$\epsilon_{1}=\epsilon_{\ominus} / 2$

First we prove $\epsilon_{1} \in \mathbb{R}$ and:

(7) $\epsilon_{1}>0$

These follow from $\epsilon_{0} \in \mathbb{R}$, (5), and elementary properties of $\mathbb{R}$. Using $\epsilon_{1} \in \mathbb{R}$ and (7), we instantiate (1) and we obtain : 


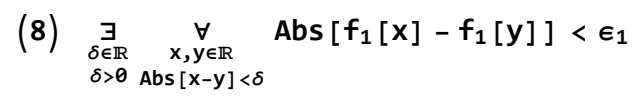

Using $\epsilon_{1} \in \mathbb{R}$ and (7), we instantiate (2) and we obtain :

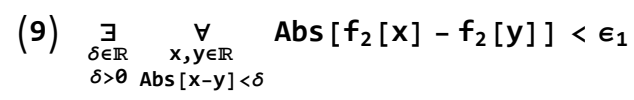

By (8) we can take $\delta_{1} \in \mathbb{R}$, such that :

(10) $\delta_{1}>0 \wedge \underset{\substack{x, y \in \mathbb{R} \\ \operatorname{Abs}[x-y]<\delta_{1}}}{\forall}$ Abs $\left[f_{1}[x]-f_{1}[y]\right]<\epsilon_{1}$

By (9) we can take $\delta_{2} \in \mathbb{R}$ such that:

(11) $\delta_{2}>\theta \wedge \underset{\substack{x, y \in \mathbb{R} \\ \operatorname{Abs}[x-y]<\delta_{2}}}{\forall} \operatorname{Abs}\left[f_{2}[x]-f_{2}[y]\right]<\epsilon_{1}$

We consider :

$\delta_{\theta}=\operatorname{Min}\left[\delta_{1}, \delta_{2}\right]$

First we prove $\delta_{0} \in \mathbb{R}$ and:

(12) $\delta_{\theta}>\theta$

These follow from $\delta_{1}, \delta_{2} \in \mathbb{R},(10.1),(11.1)$ and elementary properties of $\mathbb{R}$.

Using $\delta_{0} \in \mathbb{R}$ and (12), in order to prove (6) it suffices to prove:

(13) $\underset{\substack{x, y \in \mathbb{R} \\ \operatorname{Abs}[x-y]<\delta_{\theta}}}{\forall} \operatorname{Abs}\left[\left(f_{1}[x]+f_{2}[x]\right)-\left(f_{1}[y]+f_{2}[y]\right)\right]<\epsilon_{\theta}$

For proving (13) we take $x_{0}, y_{0} \in \mathbb{R}$ arbitrary but fixed, we assume :

(14) Abs $\left[x_{\theta}-y_{\theta}\right]<\delta_{\theta}$

and we prove :

(15) $\operatorname{Abs}\left[\left(f_{1}\left[x_{\theta}\right]+f_{2}\left[x_{\theta}\right]\right)-\left(f_{1}\left[y_{\theta}\right]+f_{2}\left[y_{\theta}\right]\right)\right]<\epsilon_{\theta}$

First we prove :

(16) Abs $\left[x_{\theta}-y_{\theta}\right]<\delta_{1} \wedge$ Abs $\left[x_{\theta}-y_{\theta}\right]<\delta_{2}$

This follows from $x_{0}, y_{0}, \delta_{\theta}, \delta_{1}, \delta_{2} \in \mathbb{R},(14)$ and elementary properties of $\mathbb{R}$.

Using $x_{0}, y_{0} \in \mathbb{R}$ and (16.1), from (10.2) we obtain:

(17) Abs $\left[f_{1}\left[x_{\theta}\right]-f_{1}\left[y_{\theta}\right]\right]<\epsilon_{1}$

Using $x_{0}, y_{0} \in \mathbb{R}$ and (16.2), from (11.2) we obtain:

(18) Abs $\left[f_{2}\left[x_{\theta}\right]-f_{2}\left[y_{\theta}\right]\right]<\epsilon_{1}$

Using elementary properties of $\mathbb{R}$ we transform (15) into:

(19) Abs $\left[\left(f_{1}\left[x_{\theta}\right]-f_{1}\left[y_{\theta}\right]\right)+\left(f_{2}\left[x_{\theta}\right]-f_{2}\left[y_{\theta}\right]\right)\right]<\epsilon_{\theta}$

Using elementary properties of $\mathbb{R}$, from (17) and (18) we obtain:

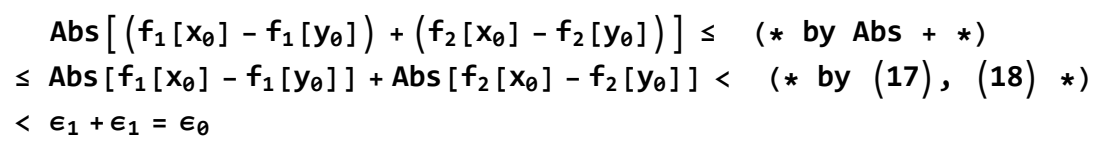

which proves the goal. 


\section{Proof discovery}

Brown text constitutes comment and is not part of the proof.

[1] Inference rule "arbitrary but fixed" : For proving an universally quantified formula with type declarations and conditions, use arbitrary but fixed constants instead of the quantified variables, assume they satisfy the type declarations and the conditions, and prove the unquantified formula.

For proving (G) we take $f_{1}, f_{2}: \mathbb{R} \rightarrow \mathbb{R}$ arbitrary but fixed, we assume :

\section{$\left(A_{1}\right) \cup\left[f_{1}\right]$}

\section{$\left(A_{2}\right) \cup\left[f_{2}\right]$}

and we prove:

(G) $U\left[f_{1}+f_{2}\right]$

Expanding the assumptions and the goal by definition. When instantiating the definition, check the type declaration and the conditions for the instantiating term.

From $\left(A_{1}\right)$, using $f_{1}: \mathbb{R} \rightarrow \mathbb{R}$, by the definition $\left(D_{1}\right)$ we obtain :

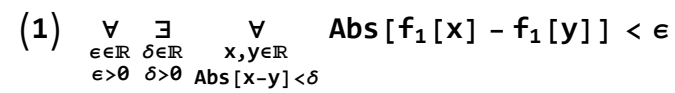

From $\left(A_{2}\right)$, using $f_{2}: \mathbb{R} \rightarrow \mathbb{R}$, by the definition $\left(D_{1}\right)$ we obtain :

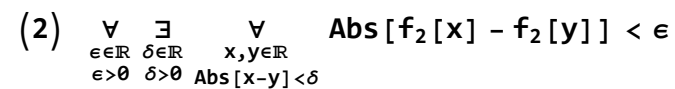

Using $f_{1}, f_{2}: \mathbb{R} \rightarrow \mathbb{R}$, by the definition $\left(D_{2}\right)$ we obtain $\left(f_{1}+f_{2}\right): \mathbb{N} \rightarrow \mathbb{R}$.

Using $\left(f_{1}+f_{2}\right): \mathbb{N} \rightarrow \mathbb{R}$, by the definition $\left(D_{1}\right)$ in order to prove $(G)$ it suffices to prove:

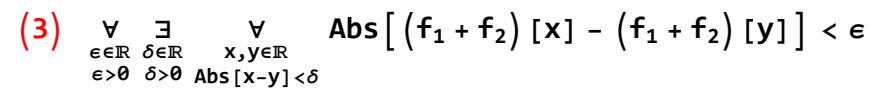

[2] Inference rule "rewriting by equality": A subterm which matches the LHS of a universally quantified equality is replaced by the instantiated RHS of the equality, after checking that the instantiating terms satisfy the type declarations and the conditions of the universal quantifiers. (The rule can be applied similarly for using the equality from right to left.)

Using $f_{1}, f_{2}: \mathbb{R} \rightarrow \mathbb{R}, x, y \in \mathbb{R}$, by $\left(D_{2}\right)$, for proving (3) it suffices to prove:

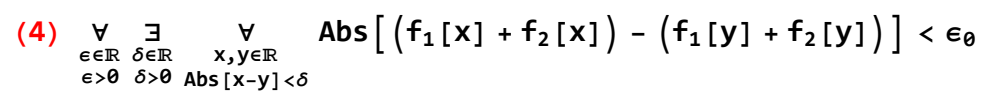

[3] Strategy "S-decomposition": When the assumptions and the goal have identical alternating quantifiers, eliminate quantifiers in parallel step by step, using a block of inference rules for each quantifier[4], [7].

[4] "S-decomposition" block of inferences for the universal quantifier: When the assumptions and the goal are universally quantified, use first the rule "arbitrary but fixed" [1] for the goal, and then the rule "instantiation" [5] for the assumptions.

Inference rule "arbitrary but fixed" [1].

For proving (4) we take $\epsilon_{0} \in \mathbb{R}$ arbitrary but fixed, we assume : 
(5) $\epsilon_{\theta}>0$

and we prove :

(6) $\underset{\delta \in \mathbb{R}}{\exists} \underset{\substack{x, y \in \mathbb{R} \\ \delta>\theta}}{\forall} \operatorname{Abs}\left[\left(f_{1}[x]+f_{2}[x]\right)-\left(f_{1}[y]+f_{2}[y]\right)\right]<\epsilon_{0}$

[5] Inference rule "instantiation": When an assumption is universally quantified, instantiate it with an appropriate ground term, after checking that the term satisfies the type declaration and the condition. In this case we instantiate (1) and (2).

[6] Method: "meta-variables": in order to find an appropriate term for the instantiation of an universal assumption[5] (or the witness term for an existential goal[10]), use a new meta-variable instead of the term (can be seen as a name for the unknown term) and continue the proof. Later in the proof find the appropriate term ("solve" the meta-variable), and check that the solution ias admissible -- contains only constants which have been already present in the proof at the moment when the meta-variable was introduced. Also check that the value satisfies the type declaration and the condition of the quantifier. In this case we use the meta-variable $\epsilon_{1}$ and the solution is found at step (20):

$\epsilon_{1}=\epsilon_{0} / 2$

First we prove $\epsilon_{1} \in \mathbb{R}$ and:

(7) $\epsilon_{1}>0$

These follow from $\epsilon_{0} \in \mathbb{R},(5)$, and elementary properties of $\mathbb{R}$.

Using $\epsilon_{1} \in \mathbb{R}$ and (7), we instantiate (1) and we obtain :

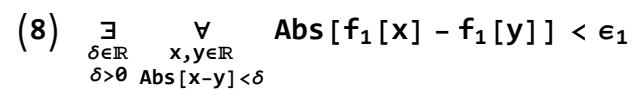

Using $\epsilon_{1} \in \mathbb{R}$ and (7), we instantiate (2) and we obtain :

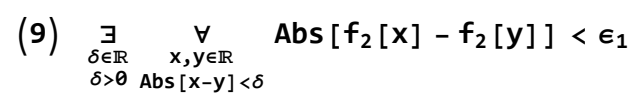

[7] "S-decomposition" block of inferences for the existential quantifier: When the assumptions and the goal are existentially quantified, use first the rule "take such a"[8] for the assumptions, and then the rule "witness"[10] for the goal.

[8] Inference rule "take such a": When an assumption is existentially quantified, use new constants instead of the quantified variables, assume they satisfy the type declarations and the conditions, and use (as assumption) the unquantified formula.

By (8) we can take $\delta_{1} \in \mathbb{R}$, such that:

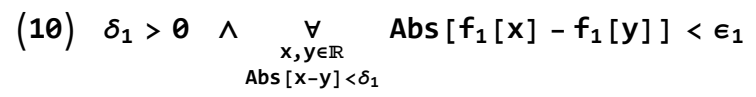

By (9) we can take $\delta_{2} \in \mathbb{R}$ such that:

(11) $\delta_{2}>0 \wedge \underset{\substack{x, y \in \mathbb{R} \\ \text { Abs }[x-y]<\delta_{2}}}{\forall}$ Abs $\left[f_{2}[x]-f_{2}[y]\right]<\epsilon_{1}$

[9] Inference rule "split assumed conjunction": The conjuncts of an assumption can be used later in the proof as individual assumptions. In this case they will be used later as (10.1), (10.2), (11.1), and 
(11.2).

[10] Inference rule "witness": When goal is existentially quantified, use an appropriate ground term as witness instead of the existential variable, checking that the term satisfies the type declaration and the condition, and then prove the unquantified formula. In this case the goal is (6).

Method: "meta-variables"[6]. We use the meta-variable $\delta_{0}$ for the witness term. The solution is found at step (16):

$\delta_{\theta}=\operatorname{Min}\left[\delta_{1}, \delta_{2}\right]$

First we prove $\delta_{0} \in \mathbb{R}$ and:

(12) $\delta_{0}>0$

These follow from $\delta_{1}, \delta_{2} \in \mathbb{R},(10.1),(11.1)$ and elementary properties of $\mathbb{R}$.

Using $\delta_{0} \in \mathbb{R}$ and (12), in order to prove (6) it suffices to prove:

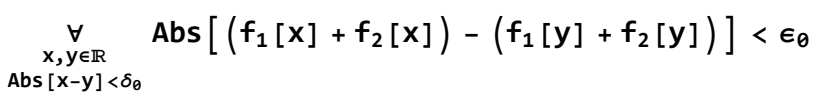

"S-decomposition" block of inferences for the universal quantifier [4].

Inference rule "arbitrary but fixed" [1].

For proving (13) we take $x_{0}, y_{0} \in \mathbb{R}$ arbitrary but fixed, we assume :

\section{(14) Abs $\left[x_{\theta}-y_{\theta}\right]<\delta_{\theta}$}

and we prove:

(15) Abs $\left[\left(f_{1}\left[x_{\theta}\right]+f_{2}\left[x_{\theta}\right]\right)-\left(f_{1}\left[y_{\theta}\right]+f_{2}\left[y_{\theta}\right]\right)\right]<\epsilon_{\theta}$

Inference rule "instantiation" [5]: we instantiate (10.2) and (11.2).

[11] Method "equal arguments to arbitrary functions": the appropriate instantiation term needed as an argument for an arbitrary function occurring in an assumption must equal a term which already occurs in the proof as the argument of this function occurring in the goal (because otherwise it will not be possible for the proof to succeed). In this case we choose $x_{0}, y_{0}$ because $f_{1}\left[x_{0}\right], f_{2}\left[x_{0}\right], f_{1}\left[y_{0}\right]$, $f_{1}\left[y_{0}\right]$ occur in the goal.

\section{First we prove :}

\section{(16) Abs $\left[x_{\theta}-y_{\theta}\right]<\delta_{1} \wedge$ Abs $\left[x_{\theta}-y_{\theta}\right]<\delta_{2}$}

[12] Method "replace equal ground terms by constants": when the same ground term occurs in several places in the formulae composing the current proof situation, we replace it by a new constant. In this case the current proof situation consists in assumption (17) and goal (19). We replace Abs $\left[\mathrm{x}_{0}-\mathrm{y}_{0}\right]$ by $\mathrm{p}$ and we transform the proof situation into:

(14.1) $\mathrm{p}<\delta_{0}$

(16.1) $\mathrm{p}<\delta_{1} \wedge \mathrm{p}<\delta_{2}$

[13] Method "solve meta-variable": use for the meta-variable a value which makes a simple proof situation to succeed, then check the appropriate conditions - see [6]. In this case the solution is $\operatorname{Min}\left[\delta_{1}, \delta_{2}\right]$. Now we go back to the proof step (12) and check that the constants $\delta_{1}, \delta_{2}$ are already present in the proof at this step, and that the solution satisfies the type declaration $(\delta \in \mathbb{R})$ and the condition $(\delta>0)$ of the main quantifier of goal (6). 
In the proof presentation this sub-proof is hidden and we will just write as argument for (16):

This follows from $x_{0}, y_{0}, \delta_{\theta}, \delta_{1}, \delta_{2} \in \mathbb{R},(14)$ and elementary properties of $\mathbb{R}$.

Inference rule "split assumed conjunction"[10]. Since (16) has been proved, we can use it now as an assumption, and split it into (16.1) and (16.2).

Using $x_{0}, y_{0} \in \mathbb{R}$ and (16.1), from (10.2) we obtain:

(17) Abs $\left[f_{1}\left[x_{\theta}\right]-f_{1}\left[y_{\theta}\right]\right]<\epsilon_{1}$

Using $x_{0}, y_{0} \in \mathbb{R}$ and (16.2), from (11.2) we obtain:

(18) Abs $\left[f_{2}\left[x_{\ominus}\right]-f_{2}\left[y_{\ominus}\right]\right]<\epsilon_{1}$

At this moment all quantifiers are eliminated by the "S-decomposition" strategy [3]. The proof situation contains only ground formulae: assumptions (17), (18) and goal (15).

Method "replace equal ground terms by constants" [12]. We replace $f_{1}\left[x_{\ominus}\right], f_{2}\left[x_{\ominus}\right], f_{1}\left[y_{\ominus}\right]$, $f_{2}\left[y_{0}\right]$ by $x_{1}, x_{2}, y_{1}, y_{2}$, respectively. The new proof situation is:

(17.1) Abs $\left[x_{1}-y_{1}\right]<\epsilon_{1}$

(18.1) Abs $\left[x_{2}-y_{2}\right]<\epsilon_{1}$

(15.1) Abs $\left[\left(x_{1}+x_{2}\right)-\left(y_{1}+y_{2}\right)\right]<\epsilon_{\theta}$

[14] Method "use constants for terms with known behaviour": replace certain ground terms occurring in the assumptions by constants, and then use equation solving, substitution, and computation in order to transform the corresponding subterms in the goal. In this case we replace $\mathrm{x}_{1}-\mathrm{y}_{1}$ and $\mathrm{x}_{2}-\mathrm{y}_{2}$ by $a_{1}, a_{2}$, respectively.

$\ln [\cdot]:=\operatorname{EqS}=\left\{\mathbf{a}_{1}=\mathbf{x} \mathbf{x}_{1}-\mathbf{y}_{1}, \mathbf{a}_{2}==\mathbf{x}_{2}-\mathbf{y}_{2}\right\} ;$

In order to update the goal we eliminate $y_{1}, y_{2}$. We can as well eliminate $x_{1}, x_{2}$, but this does not matter because the corresponding Skolem constants $\left(x_{0}, y_{0}\right)$ are introduced at the same step in the proof. (The heuristics consists in eliminating the constants corresponding to the Skolem constants introduced later in the proof, in order to maximize the chances for the admissibility of the solution.)

$\ln [\cdot]:=$ Sols $=$ First $\left[\right.$ Solve $\left[\right.$ Eqs, $\left.\left.\left\{\mathbf{y}_{1}, \mathbf{y}_{2}\right\}\right]\right]$

Out $\sigma=\left\{\mathrm{y}_{1} \rightarrow-\mathrm{a}_{1}+\mathrm{x}_{1}, \mathrm{y}_{2} \rightarrow-\mathrm{a}_{2}+\mathrm{x}_{2}\right\}$

$\ln [\cdot]:=$ Expand $\left[\left(\mathbf{x}_{1}+\mathbf{x}_{2}\right)-\left(\mathbf{y}_{1}+\mathbf{y}_{2}\right) /\right.$. Sols $]$

Out[o]= $\mathrm{a}_{1}+\mathrm{a}_{2}$

The new proof situation is:

(17.2) Abs $\left[a_{1}\right]<\epsilon_{1}$

(18.2) Abs $\left[a_{2}\right]<\epsilon_{1}$

(15.2) Abs $\left[a_{1}+a_{2}\right]<\epsilon_{\theta}$

In the proof presentation this process is hidden, we just write the expression in (16.2) by substituting back to the original terms:

Using elementary properties of $\mathbb{R}$ we transform (15) into:

(19) $\operatorname{Abs}\left[\left(f_{1}\left[x_{\theta}\right]-f_{1}\left[y_{\theta}\right]\right)+\left(f_{2}\left[x_{\theta}\right]-f_{2}\left[y_{\theta}\right]\right)\right]<\epsilon_{\theta}$ 
[15] Method "rewrite goal by inequalities": in order to prove an inequality, replace subterms by bigger subterms.

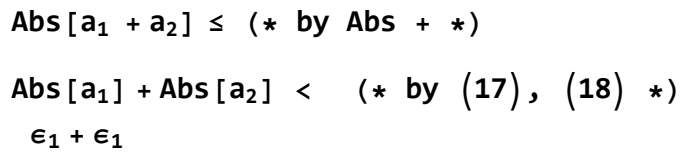

From the transformations above we have the new assumption:

\section{(20) Abs $\left[a_{1}+a_{2}\right]<2 * \epsilon_{1}$}

Method "replace equal ground terms by constants" [12]. We transform (20) and the current goal $(15.2)$ :

(20.1) $\mathrm{c}<2 * \epsilon_{1}$

$(15.3) c<\epsilon_{\theta}$

Method "solve meta-variable"[13]. In order to succeed in the proof situation above, we find $\in_{1}$ :

$\epsilon_{1}=\epsilon_{0} / 2$

Now we go back to step (7) and check that the constant $\mathbf{e}_{\boldsymbol{\theta}}$ occurring in the solving term is already present in the proof, and that the type declaration and the conditions of the universal quantifiers in (1) and (2) are satisfied.

Now the proof is finished because the main goal was proven (by an appropriate choice of a solution for the meta-variable).

In the proof presentation this process is hidden, instead the following argument is given, which is produced by substituting in the expressions above the original ground terms:

Using elementary properties of $\mathbb{R}$, from (17) and (18) we obtain:

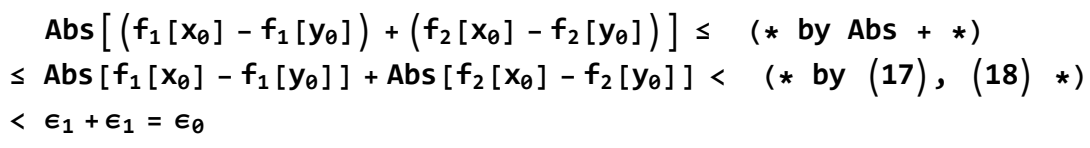

which proves the goal.

\section{Uniform Continuity of Product of Two Functions}

\section{Definitions}

Uniform continuity

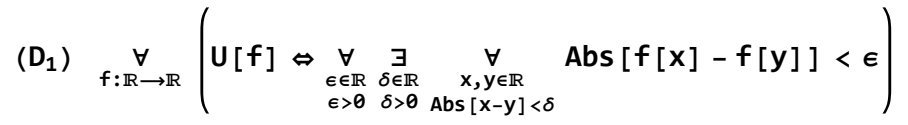

\section{Product of functions}

$\left(D_{2}\right) \underset{f_{1}, f_{2}: \mathbb{R} \rightarrow \mathbb{R}}{\forall}\left(\left(f_{1} * f_{2}\right): \mathbb{R} \rightarrow \mathbb{R} \wedge \underset{x \in \mathbb{R}}{\forall}\left(f_{1} * f_{2}\right)[x]=\left(f_{1}[x] * f_{2}[x]\right)\right)$ 


\section{Product of uniformly continuous functions is uniformly continuous}

This proof fails. In fact, the product of uniformly continuous functions is not always uniformly continuous. The failed proof attempt demonstrates the importance of checking the appropriateness of the solutions of meta-variables.

\section{Formula to prove}

(G) $\underset{\substack{f_{1}, f_{2}: \mathbb{R} \rightarrow \mathbb{R} \\ U\left[f_{1}\right], U\left[f_{2}\right]}}{\forall} U\left[f_{1}+f_{2}\right]$

\section{Proof presentation}

For proving (G) we take $f_{1}, f_{2}: \mathbb{R} \rightarrow \mathbb{R}$ arbitrary but fixed, we assume :

$\left(A_{1}\right) \cup\left[f_{1}\right]$

$\left(A_{2}\right) \cup\left[f_{2}\right]$

and we prove :

(G) $U\left[f_{1}+f_{2}\right]$

From $\left(A_{1}\right)$, using $f_{1}: \mathbb{R} \rightarrow \mathbb{R}$, by the definition $\left(D_{1}\right)$ we obtain :

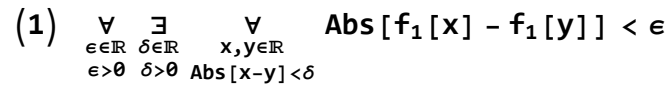

From $\left(A_{2}\right)$, using $f_{2}: \mathbb{R} \rightarrow \mathbb{R}$, by the definition $\left(D_{1}\right)$ we obtain :

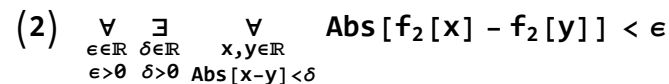

Using $f_{1}, f_{2}: \mathbb{R} \rightarrow \mathbb{R}$, by the definition $\left(D_{2}\right)$ we obtain $\left(f_{1} * f_{2}\right): \mathbb{R} \rightarrow \mathbb{R}$.

Using $\left(f_{1} * f_{2}\right): \mathbb{R} \rightarrow \mathbb{R}$, by the definition $\left(D_{1}\right)$, for proving $(G)$ it suffices to prove:

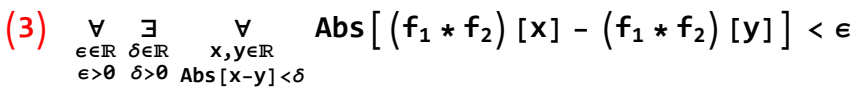

Using $f_{1}, f_{2}: \mathbb{R} \rightarrow \mathbb{R}, x, y \in \mathbb{R}$, by the definition $\left(D_{2}\right)$, for proving (3) it suffices to prove:

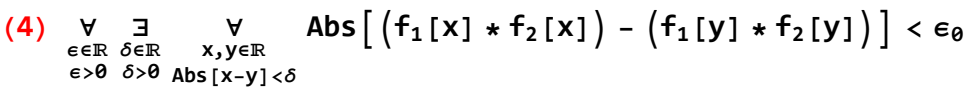

For proving (4) we take $\epsilon_{0} \in \mathbb{R}$ arbitrary but fixed, we assume :

(5) $\epsilon_{\theta}>0$

and we prove:

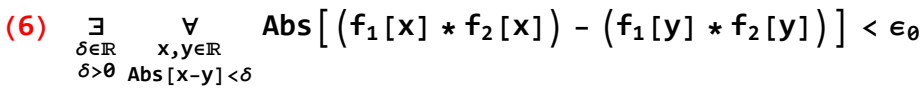

We cannot find an appropriate ground term for the instantiation of (1) and (2), thus the proof fails.

\section{Proof discovery}

Brown text constitutes comment and is not part of the proof. 
[1] Inference rule "arbitrary but fixed" : For proving an universally quantified formula with type declarations and conditions, use arbitrary but fixed constants instead of the quantified variables, assume they satisfy the type declarations and the conditions, and prove the unquantified formula.

For proving $(G)$ we take $f_{1}, f_{2}: \mathbb{R} \rightarrow \mathbb{R}$ arbitrary but fixed, we assume :

\section{$\left(A_{1}\right) \cup\left[f_{1}\right]$}

\section{$\left(A_{2}\right) \cup\left[f_{2}\right]$}

and we prove:

(G) $U\left[f_{1} * f_{2}\right]$

Expanding the assumptions and the goal by definition. When instantiating the definition, check the type declaration and the conditions for the instantiating term.

From $\left(A_{1}\right)$, using $f_{1}: \mathbb{R} \rightarrow \mathbb{R}$, by the definition $\left(D_{1}\right)$ we obtain :

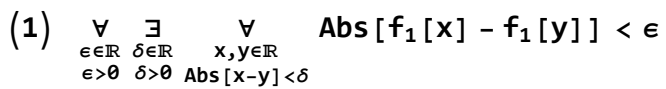

From $\left(A_{2}\right)$, using $f_{2}: \mathbb{R} \rightarrow \mathbb{R}$, by the definition $\left(D_{1}\right)$ we obtain :

(2)

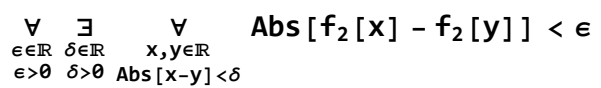

Using $f_{1}, f_{2}: \mathbb{R} \rightarrow \mathbb{R}$, by the definition $\left(D_{2}\right)$ we obtain $\left(f_{1} * f_{2}\right): \mathbb{R} \rightarrow \mathbb{R}$.

Using $\left(f_{1} * f_{2}\right): \mathbb{R} \rightarrow \mathbb{R}$, by the definition $\left(D_{1}\right)$ in order to prove $(G)$ it suffices to prove:

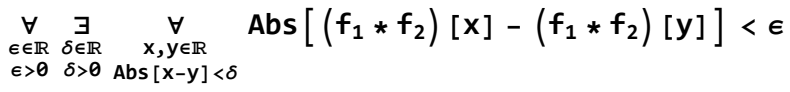

[2] Inference rule "rewriting by equality": A subterm which matches the LHS of a universally quantified equality is replaced by the instantiated RHS of the equality, after checking that the instantiating terms satisfy the type declarations and the conditions of the universal quantifiers. (The rule can be applied similarly for using the equality from right to left.)

Using $f_{1}, f_{2}: \mathbb{R} \rightarrow \mathbb{R}, x, y \in \mathbb{R}$, by $\left(D_{2}\right)$, for proving (3) it suffices to prove:

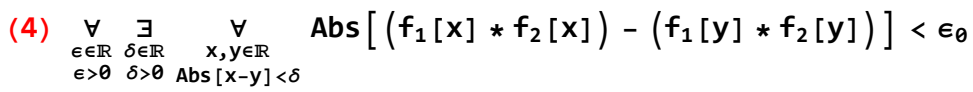

[3] Strategy "S-decomposition": When the assumptions and the goal have identical alternating quantifiers, eliminate quantifiers in parallel step by step, using a block of inference rules for each quantifier[4], [7].

[4] "S-decomposition" block of inferences for the universal quantifier: When the assumptions and the goal are universally quantified, use first the rule "arbitrary but fixed"[1] for the goal, and then the rule "instantiation"[5] for the assumptions.

Inference rule "arbitrary but fixed" [1].

For proving (4) we take $\epsilon_{0} \in \mathbb{R}$ arbitrary but fixed, we assume :

(5) $\epsilon_{\theta}>\theta$

and we prove: 


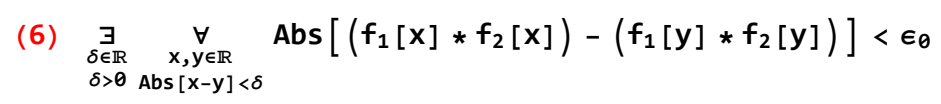

[5] Inference rule "instantiation": When an assumption is universally quantified, instantiate it with an appropriate ground term, after checking that the term satisfies the type declaration and the condition. In this case we instantiate (1) and (2).

[6] Method: "meta-variables": in order to find an appropriate term for the instantiation of an universal assumption[5] (or the witness term for an existential goal[10]), use a new meta-variable instead of the term (can be seen as a name for the unknown term) and continue the proof. Later in the proof find the appropriate term ("solve" the meta-variable), and check that the solution is admissible, namely contains only constants which have been already present in the proof at the moment when the meta-variable was introduced. (Admissibility may be achieved by reordering the steps of the proof - see proof of continuity of composition). Also check that the value satisfies the type declaration and the condition of the quantifier. In this case we use the meta-variable $\epsilon_{1}$ and the solution is found at step (19):

$\epsilon_{1}=\operatorname{Min}\left[1, \frac{\epsilon_{\theta}}{\operatorname{Abs}\left[f_{1}\left[x_{\theta}\right]\right]+\operatorname{Abs}\left[f_{2}\left[x_{\theta}\right]\right]+1}\right]$

However this solution is not appropriate for the proof, because it contains the constant $\mathbf{x}_{\boldsymbol{\theta}}$ which is not yet present in the proof. Therefore the proof fails, however we continue it below in order to show how the solution to the meta-variable is found and why it is not possible to reorder the steps of the proof in order to achieve admissibility.

First we prove $\epsilon_{1} \in \mathbb{R}$ and:

(7) $\epsilon_{1}>0$

The proof of (7) must be done after an appropriate solution to the meta-variable is found, which will not be the case, however the proof now continues.

Using $\epsilon_{1} \in \mathbb{R}$ and (7), we instantiate (1) and we obtain :
(8) $\underset{\delta \in \mathbb{R}}{\exists} \underset{\substack{x, y \in \mathbb{R} \\ \delta}}{\forall}$ $\delta>\theta$ Abs $[x-y]<\delta$
Abs $\left[f_{1}[x]-f_{1}[y]\right]<\epsilon_{1}$

Using $\epsilon_{1} \in \mathbb{R}$ and (7), we instantiate (2) and we obtain :

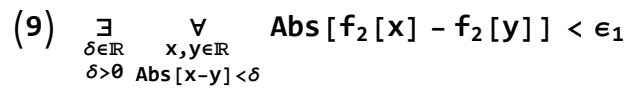

[7] "S-decomposition" block of inferences for the existential quantifier: When the assumptions and the goal are existentially quantified, use first the rule "take such a"[8] for the assumptions, and then the rule "witness"[10] for the goal.

[8] Inference rule "take such a": When an assumption is existentially quantified, use new constants instead of the quantified variables, assume they satisfy the type declarations and the conditions, and use (as assumption) the unquantified formula.

By (8) we can take $\delta_{1} \in \mathbb{R}$, such that:

(10) $\delta_{1}>0 \wedge \underset{\substack{x, y \in \mathbb{R} \\ \text { Abs }[x-y]<\delta_{1}}}{\forall}$ Abs $\left[f_{1}[x]-f_{1}[y]\right]<\epsilon_{1}$

By (9) we can take $\delta_{2} \in \mathbb{R}$ such that: 


\section{(11) $\delta_{2}>0 \wedge \underset{\substack{x, y \in \mathbb{R} \\ A b s[x-y]<\delta_{2}}}{\forall}$ Abs $\left[f_{2}[x]-f_{2}[y]\right]<\epsilon_{1}$}

[9] Inference rule "split assumed conjunction": The conjuncts of an assumption can be used later in the proof as individual assumptions. In this case they will be used later as (10.1), (10.2), (11.1), and (11.2).

[10] Inference rule "witness": When goal is existentially quantified, use an appropriate ground term as witness instead of the existential variable, checking that the term satisfies the type declaration and the condition, and then prove the unquantified formula. In this case the goal is (6).

Method: "meta-variables"[6]. We use the meta-variable $\delta_{0}$ for the witness term. The solution is found at step (16):

$\delta_{\theta}=\operatorname{Min}\left[\delta_{1}, \delta_{2}\right]$

First we prove $\delta_{0} \in \mathbb{R}$ and:

(12) $\delta_{\theta}>0$

These follow from $\delta_{1}, \delta_{2} \in \mathbb{R},(10.1),(11.1)$ and elementary properties of $\mathbb{R}$.

Using $\delta_{0} \in \mathbb{R}$ and (12), in order to prove (6) it suffices to prove:

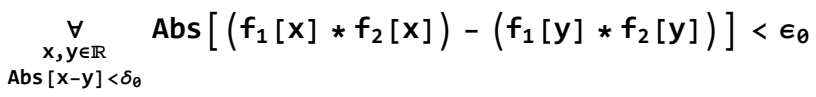

"S-decomposition" block of inferences for the universal quantifier [4].

Inference rule "arbitrary but fixed" [1]. The constant $x_{0}$ which is necessary for the instantiation at the step (7) is introduced in the proof only now, thus it cannot be used before.

For proving (13) we take $x_{0}, y_{0} \in \mathbb{R}$ arbitrary but fixed, we assume :

\section{(14) Abs $\left[x_{\theta}-y_{\theta}\right]<\delta_{\theta}$}

and we prove:

(15) Abs $\left[\left(f_{1}\left[x_{\theta}\right] * f_{2}\left[x_{\theta}\right]\right)-\left(f_{1}\left[y_{\theta}\right] * f_{2}\left[y_{\theta}\right]\right)\right]<\epsilon_{\theta}$

Inference rule "instantiation" [5]: we instantiate (10.2) and (11.2).

[11] Method "equal arguments to arbitrary functions": the appropriate instantiation term needed as an argument for an arbitrary function occurring in an assumption must equal a term which already occurs in the proof as the argument of this function occurring in the goal (because otherwise it will not be possible for the proof to succeed). In this case we choose $x_{0}, y_{0}$ because $f_{1}\left[x_{0}\right], f_{2}\left[x_{0}\right], f_{1}\left[y_{0}\right]$, $f_{1}\left[y_{0}\right]$ occur in the goal.

\section{First we prove :}

(16) Abs $\left[x_{\theta}-y_{\theta}\right]<\delta_{1} \wedge$ Abs $\left[x_{\theta}-y_{\theta}\right]<\delta_{2}$

[12] Method "replace equal ground terms by constants": when the same ground term occurs in several places in the formulae composing the current proof situation, we replace it by a new constant. In this case the current proof situation consists in assumption (17) and goal (19). We replace Abs $\left[\mathrm{x}_{0}-\mathrm{y}_{0}\right]$ by $\mathrm{p}$ and we transform the proof situation into:

$$
\begin{aligned}
& (14.1) \quad \mathrm{p}<\delta_{0} \\
& (16.1) \quad \mathrm{p}<\delta_{1} \wedge \mathrm{p}<\delta_{2}
\end{aligned}
$$


[13] Method "solve meta-variable": use for the meta-variable a value which makes a simple proof situation to succeed, then check the appropriate conditions - see [6]. In this case the solution is $\operatorname{Min}\left[\delta_{1}, \delta_{2}\right]$. Now we go back to the proof step (12) and check that the constants $\delta_{1}, \delta_{2}$ are already present in the proof at this step, and that the solution satisfies the type declaration $(\delta \in \mathbb{R})$ and the condition $(\delta>0)$ of the main quantifier of goal (6).

In the proof presentation this sub-proof is hidden and we will just write as argument for (16):

This follows from $x_{0}, y_{0}, \delta_{\theta}, \delta_{1}, \delta_{2} \in \mathbb{R},(14)$ and elementary properties of $\mathbb{R}$.

Inference rule "split assumed conjunction"[10]. Since (16) has been proved, we can use it now as an assumption, and split it into (16.1) and (16.2).

Using $x_{0}, y_{0} \in \mathbb{R}$ and (16.1), from (10.2) we obtain:

(17) Abs $\left[f_{1}\left[x_{\ominus}\right]-f_{1}\left[y_{\ominus}\right]\right]<\epsilon_{1}$

Using $x_{0}, y_{0} \in \mathbb{R}$ and (16.2), from (11.2) we obtain:

(18) Abs $\left[f_{2}\left[x_{\ominus}\right]-f_{2}\left[y_{\ominus}\right]\right]<\epsilon_{1}$

At this moment all quantifiers are eliminated by the "S-decomposition" strategy [3]. The proof situation contains only ground formulae: assumptions (17), (18) and goal (15).

Method "replace equal ground terms by constants" [12]. We replace $f_{1}\left[x_{\ominus}\right], f_{2}\left[x_{\ominus}\right], f_{1}\left[y_{\ominus}\right]$, $f_{2}\left[y_{0}\right]$ by $x_{1}, x_{2}, y_{1}, y_{2}$, respectively. The new proof situation is:

(17.1) Abs $\left[x_{1}-y_{1}\right]<\epsilon_{1}$

(18.1) Abs $\left[x_{2}-y_{2}\right]<\epsilon_{1}$

$(15.1) \operatorname{Abs}\left[\left(x_{1} * x_{2}\right)-\left(y_{1} * y_{2}\right)\right]<\epsilon_{\theta}$

[14] Method "use constants for terms with known behavior": replace certain ground terms occurring in the assumptions by constants, and then use equation solving, substitution, and computation in order to transform the corresponding subterms in the goal. In this case we replace $\mathrm{x}_{1}-\mathrm{y}_{1}$ and $\mathrm{x}_{2}-\mathrm{y}_{2}$ by $a_{1}, a_{2}$, respectively.

$\ln [e]=\operatorname{EqS}=\left\{a_{1}=\mathbf{x}_{1}-\mathbf{y}_{1}, \mathbf{a}_{2}==\mathbf{x}_{2}-\mathbf{y}_{2}\right\} ;$

In order to update the goal we eliminate $y_{1}, y_{2}$.

$\ln [\cdot]=$ Sols $=$ First $\left[\right.$ Solve $\left[\right.$ Eqs, $\left.\left.\left\{y_{1}, y_{2}\right\}\right]\right]$

oute $=\left\{\mathbf{y}_{1} \rightarrow-\mathrm{a}_{1}+\mathrm{x}_{1}, \mathrm{y}_{2} \rightarrow-\mathrm{a}_{2}+\mathrm{x}_{2}\right\}$

$\ln [e]:=$ Expand $\left[\left(\mathbf{x}_{1} * \mathbf{x}_{2}\right)-\left(\mathbf{y}_{1} * \mathbf{y}_{2}\right) /\right.$. Sols $]$

oute $=-a_{1} a_{2}+a_{2} x_{1}+a_{1} x_{2}$

The new proof situation is:

(17.2) Abs $\left[a_{1}\right]<\epsilon_{1}$

(18.2) Abs $\left[a_{2}\right]<\epsilon_{1}$

(15.2) Abs $\left[x_{1} * a_{2}+x_{2} * a_{1}+a_{1} * a_{2}\right]<\epsilon_{\theta}$

[15] Method "rewrite goal by inequalities": in order to prove an inequality, replace subterms by bigger subterms. 


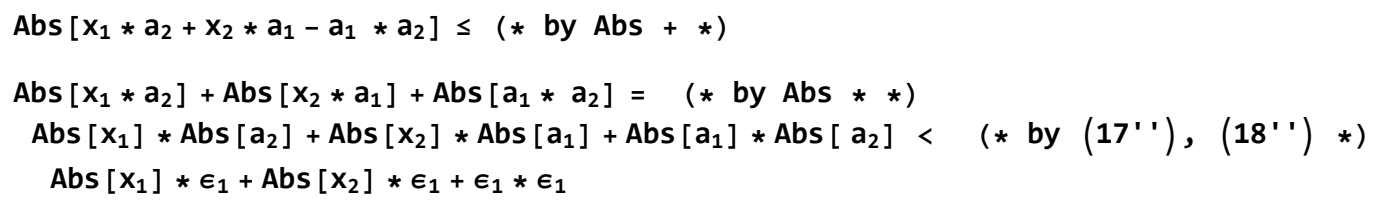

[16] Method "bound the epsilon-bound": the conditional bound for the absolute value (as here $\in_{1}$ ) can be bounded by any positive value, because the original formula will still be satisfied if the bound is smaller. In this case we use the bound 1 , and we add $\epsilon_{1} \leq 1$ to the current goal.

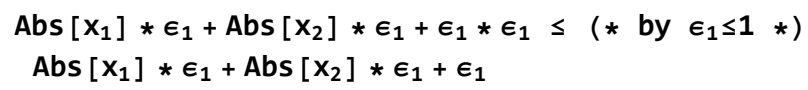

[17] Method "find meta-variable by factoring": transform the subterm of the goal containing the meta-variable in an expression which can be factored.

$\operatorname{Abs}\left[x_{1}\right] * \epsilon_{1}+\operatorname{Abs}\left[x_{2}\right] * \epsilon_{1}+\epsilon_{1}=\epsilon_{1} *\left(\operatorname{Abs}\left[x_{1}\right]+\operatorname{Abs}\left[x_{2}\right]+1\right)$

From the transformations above we have the new assumption:

(19) Abs $\left[x_{1} * a_{2}+x_{2} * a_{1}-a_{1} * a_{2}\right]<\epsilon_{1} *\left(\operatorname{Abs}\left[x_{1}\right]+\operatorname{Abs}\left[x_{2}\right]+1\right)$

Method "replace equal ground terms by constants" [12]. We transform (19) and the current goal $\left(15^{\prime \prime}\right)$ :

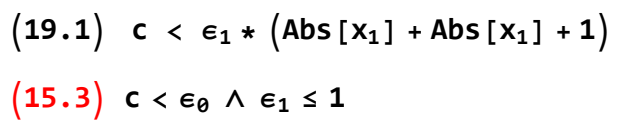

Now we go back to step ( 7$)$ and check that the constants $\left(e_{\theta}, x_{\theta}, f_{1}, f_{2}\right)$ occurring in the solving term are already present in the proof, and that the type declaration and the conditions of the universal quantifiers in (1) and (2) are satisfied. The constant $x_{\theta}$ is not present in the proof at step (7), because it is introduced only at step (14).

We try to reorder the steps of the proof in order to achieve admissibility. For this we introduce an ordering on the set of Skolem constants $\left\{\epsilon_{0}, \delta_{1}, \delta_{2}, x_{0}, y_{0}\right\}$ and metavariables $\left\{\epsilon_{1}, \delta_{0}\right\}$, that sets as smaller an element which must be generated earlier than another in the proof. Thus we have:

$a \prec b$ iff (( $a$ occurs in the formula from which $b$ is created) or ( $a$ occurs in the solution for $b)$ )

The first criterion gives us the following constraints:

$\epsilon_{1}<\delta_{1}$ formulae $(8)(10)$

$\epsilon_{1}<\delta_{2}$ formulae 9$)(11)$

$\epsilon_{\theta}<\delta_{\theta}$ formulae 11$)(13)$

$\epsilon_{\theta}, \delta_{\theta}<\mathbf{x}_{\theta}$, ye $_{\theta}$ formulae $(13)(14)(15)$

The second criterion gives us the following constraints:

$\delta_{1}, \delta_{2}<\delta_{\theta}$ solution $\delta_{\theta}$

$\mathrm{x}_{\ominus}<\epsilon_{1}$ solution $\epsilon_{1}$

We can construct the cycle :

$\epsilon_{1}<\delta_{1}<\delta_{0}<x_{0}<\epsilon_{1}$

thus it is impossible to find an ordering that satisfies all the constraints.

Therefore the proof fails. 
Note that we could proceed after step (15') alternatively: In order to update the goal we eliminate $x_{1}, x_{2}$ :

$\ln [\cdot]:=$ Sols $=$ First $\left[\right.$ Solve $\left.\left[\mathrm{Eqs},\left\{x_{1}, x_{2}\right\}\right]\right]$

Out[ $]=\left\{\mathrm{x}_{1} \rightarrow \mathrm{a}_{1}+\mathrm{y}_{1}, \mathrm{x}_{2} \rightarrow \mathrm{a}_{2}+\mathrm{y}_{2}\right\}$

$\ln [\cdot]:=$ Expand $\left[\left(\mathbf{x}_{1} * \mathbf{x}_{2}\right)-\left(\mathbf{y}_{1} * \mathbf{y}_{2}\right) /\right.$. Sols $]$

out $[\cdot]=a_{1} a_{2}+a_{2} y_{1}+a_{1} y_{2}$

We will find now in a similar way a solution for the meta-variable $\epsilon_{1}$ which contains $y_{0}$, which is also not acceptable because this constant is also introduced too late in the proof, and we can see above that a cycle can be constructed with $\mathrm{x}_{\theta}<\epsilon_{1}$ also.

\section{Continuity of Composition of Functions}

Continuity of a function (on $\mathbb{R}$ ):

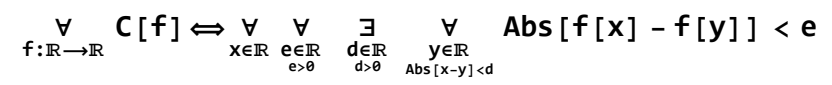

Composition of functions:

(D2) $\underset{f, g: \mathbb{R} \rightarrow \mathbb{R}}{\forall} f \circ g: \mathbb{R} \rightarrow \mathbb{R} \wedge \underset{x \in \mathbb{R}}{\forall}(f \circ g)[x]=f[g[x]]$

We prove:

$\underset{\substack{f, g: \mathbb{R} \rightarrow \mathbb{R} \\ \mathrm{C}[f],[\mathrm{g}]}}{\forall} \mathrm{C}[\mathrm{f} \circ \mathrm{g}]$

Brown text constitutes comment and is not part of the proof.

1. The steps concerning arbitrary but fixed $\mathrm{f}, \mathrm{g}: \mathbb{R} \longrightarrow \mathbb{R}$, decomposition of goal, and expansion by definition are similar to the other proofs. After these we have the proof situation:

Assumptions:

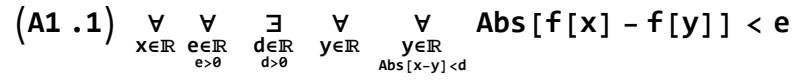

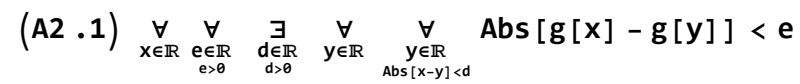

Goal:

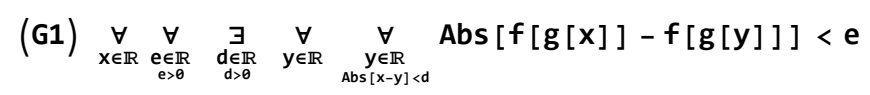

2. For proving (G1) we take a.b.f. $\mathrm{x}_{\theta} \in \mathbb{R}$ and we prove :

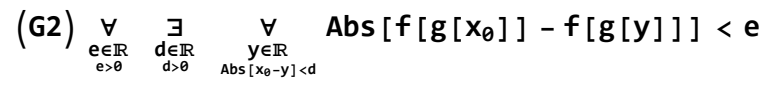

3. Instantiate (A1.1) with $\mathrm{g}\left[\mathrm{x}_{\theta}\right]$ and $(\mathrm{A} 2.1)$ with $\mathrm{x}_{\theta}$.

(heuristics: equal arguments to arbitrary functions) :

Check $x_{\theta}, g\left[x_{\theta}\right]$ in $\mathbb{R}:$ yes by steps 1 and 2 . 


$$
\begin{aligned}
& (A 1.2) \underset{\substack{e \in \mathbb{R} \\
e>\theta}}{\forall} \underset{\substack{d \in \mathbb{R} \\
d>\theta}}{\exists} \underset{\substack{y \in \mathbb{R} \\
A b s\left[g\left[x_{\theta}\right]-y\right]<d}}{\forall} \text { Abs }\left[f\left[g\left[x_{\theta}\right]\right]-f[y]\right]<e \\
& (A 2.2) \underset{\substack{e \in \mathbb{R} \\
e>\theta}}{\forall} \underset{\substack{d \in \mathbb{R} \\
d>\theta}}{\exists} \underset{\substack{y \in \mathbb{R} \\
A b s\left[x_{0}-y\right]<d}}{\forall} \text { Abs }\left[g\left[x_{\theta}\right]-g[y]\right]<e
\end{aligned}
$$

4. For proving (G2) we take a.b.f. $e_{\ominus} \in \mathbb{R}$ and we prove :

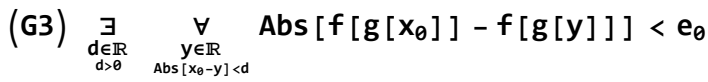

Some of the steps below use the technique of metavariables : the prover cannot determine the instantiation term or the witness at this moment, but one uses a metavariable as the name of the unknown term and the prover finds the appropriate term (solution) later in the proof. After this it is checked that the solutions are admissible (all Skolem constants present in the solution have to be already present in the proof), as well as the type and the condition.

The ordering of the steps 4 to 8 cannot be determined at this moment (only that 6 comes after 5 and 8 comes after 7). They can be generated in any order, and in case of incorrect order they will be reordered as explained after the end of the proof.

5. Instantiate (A1 .2) with $e_{1}^{*}$ (determined as $e_{\ominus}$ at step 13)

Check solutions admisible: yes ( $\mathrm{e}_{\ominus}$ introduced at step 4.)

Check $e_{0} \in \mathbb{R}, e_{0}>0$ : yes by step 4 .

(A1 .3) $\underset{\substack{y \in \mathbb{R} \\ A b s\left[g\left[x_{\theta}\right]-y\right]<d}}{\forall}$ Abs $\left[f\left[g\left[x_{\theta}\right]\right]-f[y]\right]<e_{1}^{*}$

6. By $(A 1.3)$ take $d_{1} \in \mathbb{R}, d_{1}>0$, such that :

$(A 1.4) \underset{\substack{y \in \mathbb{R} \\ \operatorname{Abs}\left[g\left[x_{\theta}\right]-y\right]<d_{1}}}{\forall}$ Abs $\left[f\left[g\left[x_{\ominus}\right]\right]-f[y]\right]<e_{1}^{*}$

7. Instantiate (A2 . 2) with $\mathrm{e}_{2}^{*}$ (determined as $\mathrm{d}_{1}$ at step 12 ) Check solutions admisible : yes ( $d_{1}$ introduced at step 6 ) . Check $d_{1} \in \mathbb{R}, d_{1}>0$ : yes by step 6 .

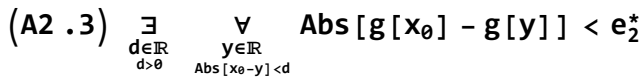

8. By $(A 2.3)$ take $d_{2} \in \mathbb{R}, d_{2}>0$, such that :

$(A 2.4) \underset{\substack{y \in \mathbb{R} \\ A b s\left[x_{0}-y\right]<d_{2}}}{\forall}$ Abs $\left[g\left[x_{\theta}\right]-g[y]\right]<e_{2}^{*}$

9. For proving (G3) is suffices to prove (witness $d_{0}^{*}$ determined as $d_{2}$ at step 11 ).

Check solution admissible: yes, $d_{2}$ introduced at step 8 . 
Check $d_{2} \in \mathbb{R}, d_{2}>0$ : yes, see step 8 .

(G4) $\underset{\substack{y \in \mathbb{R} \\ A b s\left[x_{\theta}-y\right]<d_{\theta}^{*}}}{\forall}$ Abs $\left[f\left[g\left[x_{\theta}\right]\right]-f[g[y]]\right]<e_{\ominus}$

10. For proving (G4) we take $y_{0} \in \mathbb{R}$ arbitrary but fixed, we assume :

(A3) Abs $\left[x_{\theta}-y_{\theta}\right]<d_{\theta}^{*}$

and we prove:

(G5) Abs $\left[f\left[g\left[x_{\theta}\right]\right]-f\left[g\left[y_{\theta}\right]\right]\right]<e_{\theta}$

11. Instantiate (A2 .4) with yo (because this occurs as argument in the goal) . Check $y_{\ominus} \in \mathbb{R}:$ Yes - see steps 9 and 1 .

By checking the condition of the quantifier we obtain the solution for $\mathrm{d}_{\ominus}^{*}$.

Check condition : Abs $\left[x_{\theta}-y_{\theta}\right]<d_{2}$. Yes, by $(A 3)$, if we take $d_{\theta}^{*} \rightarrow d_{2}$.

(A2 .5) Abs $\left[g\left[x_{\theta}\right]-g\left[y_{\theta}\right]\right]<e_{2}^{*}$

12. Instantiate (A1 .4) with $\mathrm{g}\left[\mathrm{y}_{0}\right]$ (because this occurs as argument in the goal) . Check $g\left[\mathrm{y}_{0}\right] \in \mathbb{R}$. Yes - see steps 9 and 1 .

Check condition : Abs $\left[g\left[x_{\ominus}\right]-g\left[y_{\ominus}\right]\right]<d_{1}$. Yes, by $(A 2.5)$, if we take $e_{2}^{*} \rightarrow d_{1}$.

(A1 .5) Abs $\left[f\left[g\left[x_{\theta}\right]\right]-f\left[g\left[y_{\theta}\right]\right]\right]<e_{1}^{*}$

13. In (A1.5) we set $\mathrm{e}_{1}^{*} \longrightarrow \mathrm{e}_{\ominus}$ and we obtain the goal: success.

The the order in which the metavariables and the Skolem constants are introduced in the proof is constrained by the inference steps: $e_{1}^{*} \prec d_{1}$ (because step 6 generates $d_{2}$ from an formula which contains $e_{1}^{*}$ ) and similarly for $e_{2}^{*} \prec d_{2}$ (step 8), and it is also constrained by the occurence of the skolem constants in the solutions to the metavariables: $d_{2} \prec d_{0}^{*}, d_{1} \prec e_{2}^{*}, e_{0} \prec e_{1}^{*}$.

These constraints must be observed by a final ordering, in this case we have:

$e_{0} \prec e_{2}^{*} \prec d_{2} \prec e_{1}^{*} \prec d_{1} \prec d_{0}^{*}$ and the inference steps are ordered according to this.

If such a final ordering cannot be found, then the proof fails. 